\title{
Similarity in fuzzy systems
}

\author{
Asim Pal ${ }^{*}{ }^{\dagger}$, Banibrata Mondal ${ }^{\dagger}$, Namrata Bhattacharyya ${ }^{\dagger}$ and Swapan Raha $^{\dagger}$
}

\author{
*Correspondence: \\ itsasimpal@rediffmail.com \\ ${ }^{\dagger}$ Equal contributors \\ Department of Mathematics, \\ Visva-Bharati, Santiniketan, 731235, \\ India
}

\begin{abstract}
This paper proposes to study the concept of similarity and its use in the design of fuzzy system. The concept of similarity relation is effectively used in fuzzification of crisp values. Similarity index is used in measuring approximate (graded) equality of fuzzy sets over a given universe of discourse. It is proposed to use such an index in modifying a fuzzy relation. Different similarity measures in the literature are elucidated, and a comparative study between different pairs of fuzzy sets is presented. One of these similarity measures has been used successfully in rule selection and modification of a fuzzy relation. In the process, a number of modification schemes based on different logic have been extensively studied for different reasoning mechanisms based on the same data and results are tabulated. A specificity-based approach to defuzzification is also presented, which is found to be suitable for similarity-based fuzzy systems. The results are illustrated with the behaviour of a direct current (DC) shunt motor.
\end{abstract}

Keywords: Approximate reasoning; Similarity; Similarity-based reasoning

\section{Introduction}

A system is defined as an integrated set of interacting elements/objects/components that accomplish a defined objective. Usually, the description of a system can be made at different levels of details. Any subsystem may, itself, be considered as a system consisting of subsystems at a lower level of detail. Human beings reason consistently from incomplete/imprecise knowledge of a system with reasonably good results. For instance, control is often exercise by a human operator who has the requisite skill to control the system successfully but cannot explain sufficiently well how he does it. Often, it becomes difficult to find even a mathematical model for the same. The tentative nature of human thinking involves frequently imprecise concepts (qualitative statements made on the input-output behaviour of such a system) which may not even have an underlying metric. Therefore, reasoning mechanisms should be modified to include provisions for handling such imprecisions in the system.

One may represent human expertise in the form of 'if statement1, then statement2'. This deductive form is commonly referred to as a rule - with statement 1 as antecedent and statement 2 as its consequence. A system, whose behaviour is described with a number of such rules, is referred to as a rule-based system. It expresses typically an inference in such a way that, with a known fact and a given rule, one can infer/derive a piece of information on the behaviour of the system which is referred to as the conclusion. This form of knowledge representation (processing) is found to be quite appropriate in expressing human understanding in a natural language of communication.

(c) 2014 Pal et al.; licensee Springer. This is an Open Access article distributed under the terms of the Creative Commons

Attribution License (http://creativecommons.org/licenses/by/2.0), which permits unrestricted use, distribution, and reproduction in any medium, provided the original work is properly credited. 
A collection of imprecise information given by human experts often forms the basis of a fuzzy system. The task of a fuzzy system is to exploit experts' knowledge and model the world with it. A fuzzy system reasons with its knowledge. A fuzzy rule-based system is one of the most important areas of research. It is a dominating platform for the development of a precise mathematical model for an imprecisely known system. Many believe that human beings take a similar approach to perceive the world around them in a robust way. In the real world, almost everything is incompletely defined. A fuzzy rule-based system is, therefore, expected to achieve a performance better than any crisp model in dealing with ambiguity, incompleteness and imprecision. A fuzzy rule-based system consists of a set of fuzzy If-THEN rules together with an inference engine, a fuzzifier and a defuzzifier.

Different patterns of reasoning in human beings indicate a need for similarity matching in situations where there are no directly applicable knowledge to come up with a plausible conclusion. In such cases, the confidence in a conclusion may be determined, based on a degree of similarity between the fact(s) and the antecedent of a rule. In order to capture this, our model should have the required flexibility. Specifically, we need means to handle graded information on the one hand and the concept of similarity on the other hand. Conventional approximate reasoning does not consider the concept of similarity measure in deriving a consequence. Existing similarity-based reasoning methods modify the consequence of a rule based on a measure of similarity, thereby making the consequence independent of the conditionals. To satisfy both requirements simultaneously, we need to integrate conventional approximate reasoning and similarity-based reasoning for an adequate theory of similarity-based approximate reasoning.

The objectives of the paper are to study the different modules of a fuzzy system and to observe the role of similarity and subsequently the possibility of a step-by-step introduction of the concept of similarity into approximate reasoning methodology which forms its core.

A formal method of fuzzification is presented in this paper. A thorough exposition on the use of different translating rules is considered. The modification mechanism will be such that every change in the conditional (general) statement and in the fact is incorporated in the fuzzy relation between the variables defining the condition. This procedure ensures the deduction/inference as a function of the concerned change. The more the change, the less specific will be the conclusion. A proposal to formulate different schema for the modification of the conditional fuzzy relation is presented. This paper proposes to demonstrate how one can avoid the use of certainty factor concept for rule misfiring. We thus have to modify the inference mechanism in a way such that a significant change will make the conclusion less specific. This can be done if an expansion type of inference scheme is chosen. Explicitly, when the similarity value becomes low, i.e. when the change is significant, the reasoning process is such that the inference becomes unknown. At the same time, when there is no change, i.e. a perfectly matching case, it is possible to derive the expected consequence (the consequence of the condition in a rulebased system). In other cases, the consequence will be no better than what the condition allows. Different interesting results in this direction are discussed extensively. It seeks to show that the concept of specificity measure of fuzzy sets is inherent in such similaritybased approximate reasoning methodology. Examples are considered to demonstrate the computations under the procedure. A comparison of the result with other existing approaches to similarity-based reasoning and Zadeh's compositional rule of inference is 
also presented. Defuzzification, a basic operation, used in the development of fuzzy system is discussed in the light of new similarity-based approximate reasoning mechanism. A new scheme for defuzzification, suitable for similarity-based approximate reasoning, is defined. This defuzzification method will then be used in problems of classification, diagnosis and control. Simulation is performed with some real data and results are tabulated.

The paper consists of seven sections. The introductory section is followed by a discussion on the similarity relation which again is followed by a proposal for constructing such an equivalence relation induced by fuzzy sets and its subsequent use in fuzzification in the section 'Similarity relation - fuzzification'. Similarity measure of two fuzzy sets is considered in the section 'Similarity measure - inference'. Approximate reasoning is discussed in the context of similarity. Different schemes are presented and examples considered to illustrate the problem. The section 'Specificity measure - defuzzification' is devoted to defuzzification of fuzzy outputs. Specificity measure of fuzzy sets can be used to determine the anxiety in decision making. A new defuzzification scheme is defined that works on specificity measures of fuzzy sets. Application of the same in different models are presented in the section 'Application in different models' followed by result of a case study on a direct current (DC) shunt motor. The paper is concluded in the section 'Conclusions'. A list of references is provided in the last section.

\section{Similarity relation - fuzzification}

Similarity is an important concept for which a crisp model is often found to be inadequate. In [1], the authors showed that the notion of membership is a gradual property of fuzzy sets. They have considered a fuzzy equivalence relation to describe the similarity between elements of a fuzzy set. There, they showed how a crisp set induced a fuzzy set as its extensional hull with respect to a fuzzy equivalence relation. Assigning similarity modelled by a fuzzy equivalence relation as the basis, fuzzy sets were viewed as induced concept. Two elements cannot be distinguished by a fuzzy set if they are both either elements of the same set or its complement [2]. They have shown how membership functions of fuzzy sets can be calculated from the fuzzy equivalence relation as in the following:

Definition 1. A fuzzy equivalence relation (with respect to the conjunction operation $*$, here, a t-norm) on the set $U$ is a mapping $E: U \times U \longrightarrow[0,1]$ satisfying
(E1) $E(u, u)=1, u \in U$
(reflexivity)
(E2) $E\left(u_{1}, u_{2}\right)=E\left(u_{2}, u_{1}\right), u_{1}, u_{2} \in U$
(symmetry)
(E3) $E\left(u_{1}, u_{2}\right) * E\left(u_{2}, u_{3}\right) \leq E\left(u_{1}, u_{3}\right), u_{1}, u_{2}, u_{3} \in U$ (transitivity).

where $[0,1]$ is the unit interval with the usual ordering. Sometimes, $E$ is also called a similarity relation [1]. Contextually, some definitions and theorems cited in [1] are hereby recalled.

Definition 2. A fuzzy set $A \in[0,1]^{U}$ is called extensional with respect to (w.r.t.) the fuzzy equivalence relation $E$ on $U$ if and only if $\mu_{A}\left(u_{1}\right) * E\left(u_{1}, u_{2}\right) \leq \mu_{A}\left(u_{2}\right)$ holds for all $u_{1}, u_{2} \in U$. 
Definition 3. Let $E$ be a fuzzy equivalence relation on $U$ and let $A \in[0,1]^{U}$.

The fuzzy set $\hat{A}=\bigcap\{B \mid A \subseteq B$ and $B$ is extensional w.r.t. $E\}$ is called the extensional hull of $A$ w.r.t. $E$.

Theorem 1. $\hat{A}=\bigcup\left\{\mu_{A}\left(u_{1}\right) * E\left(u_{1}, u_{2}\right) \mid u_{1}, u_{2} \in U\right\}$.

Theorem 2. Let $\mathcal{F} \subseteq[0,1]^{U}$ be a collection of fuzzy sets and $\leftrightarrow:[0,1] \times[0,1] \rightarrow[0,1]$ be a biimplication. Then,

$$
E_{\mathcal{F}}\left(u_{1}, u_{2}\right)=\bigwedge_{A \in \mathcal{F}}\left(\mu_{A}\left(u_{1}\right) \leftrightarrow \mu_{A}\left(u_{2}\right)\right)
$$

is the coarsest fuzzy equivalence relation on $U$ such that all fuzzy sets in $\mathcal{F}$ are extensional w.r.t. $E_{\mathcal{F}}$.

$E_{\mathcal{F}}$ is reflexive, symmetric and satisfy transitivity relation. The fuzzy equivalence relation (1) can be interpreted in the following way - two elements 'cannot be distinguished by a (fuzzy) set' if they are both elements of the same set or its complement, but not one in the set and the other one in its complement. Thus, $\mu_{A}\left(u_{1}\right) \leftrightarrow \mu_{A}\left(u_{2}\right)$ represents the degree to which the elements $u_{1}$ and $u_{2}$ cannot be distinguished by the fuzzy set $A$. Therefore, $E_{\mathcal{F}}\left(u_{1}, u_{2}\right)$ is the degree to which $u_{1}$ and $u_{2}$ cannot be distinguished by the set $\mathcal{F}$ of fuzzy sets.

We develop a new fuzzy equivalence relation on a universe of discourse $U$, which is generated by a family $\mathcal{F}$ of fuzzy subsets of $U$. Accordingly, we define a fuzzy relation $E$ as given below:

$$
E\left(u_{1}, u_{2}\right)=1-\sqrt{\frac{\Sigma_{A}\left(\mu_{A}\left(u_{1}\right)-\mu_{A}\left(u_{2}\right)\right)^{2}}{n}},
$$

where $n$ is the number of fuzzy sets.

Theorem 3. The fuzzy relation defined in (2) is a fuzzy equivalence relation.

From this fuzzy equivalence relation $E\left(u_{1}, u_{2}\right)$, we can fuzzify any point ' $a$ ' on the universe of discourse $U$ by setting

$$
\begin{aligned}
\mu_{a}\left(u_{1}\right) & =E\left(u_{1}, a\right), & & \text { in an interval } a-\delta \leq u_{1} \leq a+\delta, \delta>0, \\
& =0, & & \text { otherwise. }
\end{aligned}
$$

Observation: $\mu_{a}\left(u_{1}\right)$ is extensional with respect to the fuzzy equivalence relation $E$ as

$$
\begin{aligned}
\mu_{a} & \left(u_{1}\right) * E\left(u_{1}, u_{2}\right) \\
& =E\left(u_{1}, a\right) * E\left(u_{1}, u_{2}\right) \\
& =E\left(a, u_{1}\right) * E\left(u_{1}, u_{2}\right) \leq E\left(a, u_{2}\right) \\
& =E\left(u_{2}, a\right)=\mu_{a}\left(u_{2}\right) .
\end{aligned}
$$

Thus, given a fuzzy equivalence relation and a crisp point ' $a$ ', we can define (generate) a fuzzy set about the point ' $a$ '. This is called fuzzification and plays an important role in the design of fuzzy systems. We illustrates this fuzzification with the following algorithm. 


\section{Algorithm FUZZ: fuzzification}

Step 1. Given $n$ fuzzy sets $A_{1}, A_{2}, \ldots, A_{n}$ defined over some universe of discourse $U$ and $a \in U$.

Step 2. Construct a fuzzy equivalence relation $E\left(u_{1}, u_{2}\right)$ from $A_{1}, A_{2}, \ldots, A_{n}$ using (2).

Step 3. Set $\delta>0$. Define a fuzzy set about the point $a \in U$ from the fuzzy equivalence relation $E\left(u_{1}, u_{2}\right)$ by

$$
\begin{array}{rlrl}
\mu_{a}\left(u_{1}\right) & =E\left(u_{1}, a\right), & \text { in the interval } a-\delta \leq u_{1} \leq a+\delta, \delta>0 \\
& =0, \quad \text { otherwise. }
\end{array}
$$

Example 1. Let the domain set be $U=\{0.0,0.1,0.2,0.3,0.4, \ldots, 9.9,10.0\}$.

Let $A_{r}$ be the fuzzy sets corresponding the points $r=0.0,0.5,1.0,1.5, \ldots, 10.0$, i.e. the set of fuzzy sets $\left\{\mu_{A_{i}} \mid i \in R\right.$, a finite index set $\}$ where

$$
\mu_{A_{i}}(u)=1-\min \{|u-i|, 1\} \quad[1] .
$$

Now, a fuzzy equivalence relation induced by the fuzzy sets as given in Figure 1 and using the algorithm FUZZ is given in Figure 2.

Choosing $\delta=5.0$, we find the extensional hulls of the crisp values 5.0 and 7.5 with respect to this fuzzy equivalence relation are the fuzzy sets

$$
\begin{aligned}
& \mu_{5.0}(u)=E(u, 5.0) \text { if } 5.0-5 \leq u \leq 5.0+5, \text { otherwise } \mu_{5.0}=0 \text { and } \\
& \mu_{7.5}(u)=E(u, 7.5) \text { if } 7.5-5 \leq u \leq 7.5+5, \text { otherwise } \mu_{7.5}=0 .
\end{aligned}
$$

\section{Similarity measure - inference}

The similarity between two objects suggests the degree to which the properties of one may be inferred from those of the other. In this section, we present some similarity measures that exist in the literature and their performances are studied. At the end, we investigate similarity-based fuzzy reasoning techniques with the best of these measures.

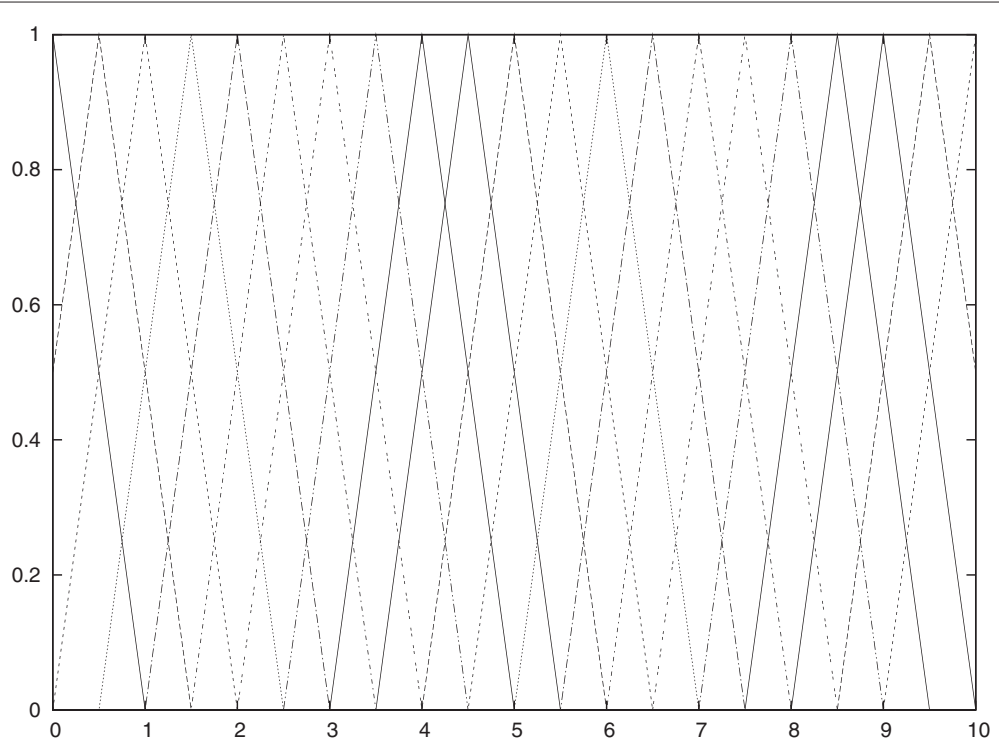

Figure 1 A typical fuzzy partition. A number of fuzzy sets on a domain draw a fuzzy partition. 


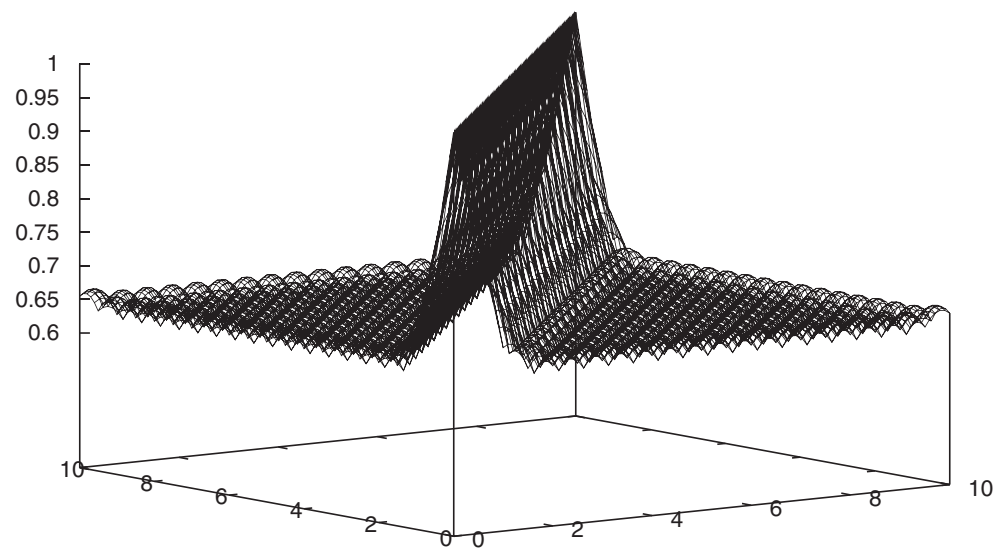

Figure 2 An equivalence relation. An equivalence relation from the fuzzy partition.

\section{Similarity measure}

A similarity matching degree can be defined from the distance functions according to the following: $S(\bullet, \bullet)=1-d(\bullet, \bullet)$. The most important class of distance function is the Minkowski's $r$-metric. Another important class of distance functions is given by the Hausdorff metric. It is a generalisation of the distance between two points in a metric space to two compact non-empty subsets of the space [3]. A set theoretic approach to a family of similarity functions can be given by $S(A, B)=\theta f(A \cap B)-\alpha f(A-B)-\beta f(B-A)$ for some function $f$ and parameters $\theta, \alpha, \beta \geq 0$ [4].

Let us assume the universe of discourse $\bar{U}$ to be a finite set and $A=\sum_{u \in U}\left\{\mu_{A}(u) / u\right\}$, $B=\sum_{u \in U}\left\{\mu_{B}(u) / u\right\}$ be two fuzzy sets defined over $U$. A similarity index between the pair $\{A, B\}$ is denoted as $S(A, B ; U)$ or simply $S(A, B)$. In the following, a number of existing similarity measures are listed from the literature.

1. $\operatorname{sim} 1=1-\left(\frac{\Sigma_{u}\left|\mu_{A}(u)-\mu_{B}(u)\right|^{q}}{n}\right)^{\frac{1}{q}}[5,6]$, where $n$ is the cardinality of the universe of discourse and $q$ is the family parameter.

2. $\operatorname{sim} 2=1-\max _{u \in U}\left(\left|\mu_{A}(u)-\mu_{B}(u)\right|\right)$ [7].

3. $\operatorname{sim} 3=1-\frac{\Sigma_{u \in U}\left|\left(\mu_{A}(u)-\mu_{B}(u)\right)\right|}{\Sigma_{u \in U}\left(\mu_{A}(u)+\mu_{B}(u)\right)}$ [7].

4. $\operatorname{sim} 4=1-\frac{1}{n} \Sigma_{u \in U}\left|\mu_{A}(u)-\mu_{B}(u)\right|$ [7].

5. $\operatorname{sim} 5=\frac{\max _{u \in U}\left\{\mu_{A}(u) \wedge \mu_{B}(u)\right\}}{\max _{u \in U}\left\{\max \left(\mu_{A}(u)\right), \max \left(\mu_{B}(u)\right)\right\}}$ [6].

6. $\operatorname{sim} 6=\frac{\max \left\{\mu_{A}(u) \cdot \mu_{B}(u)\right\}}{\max \left\{\Sigma_{u \in U} \mu_{A}^{2}(u), \Sigma_{u \in U} \mu_{B}^{2}(u)\right\}}$ [7].

7. $\quad \operatorname{sim} 7=\frac{\min \left(\mu_{A}(u), \mu_{B}(u)\right)}{\max \left(\mu_{A}(u), \mu_{B}(u)\right)}$ [7].

8. $\quad \operatorname{sim} 8=\frac{1}{n} \Sigma_{u \in U} \frac{\min \left(\mu_{A}(u), \mu_{B}(u)\right)}{\max \left(\mu_{A}(u), \mu_{B}(u)\right)}$ [8].

9. $\operatorname{sim} 9=\max _{u \in U}\left\{\min \left(\mu_{A}(u), \mu_{B}(u)\right)\right\}$ [9].

10. $\operatorname{sim} 10=\frac{C(A, B)}{\sqrt{T(A), T(B)}}[8]$, where $T(A)=\Sigma_{i=1}^{n}\left(\mu_{A}\left(u_{i}\right)^{2} \cdot\left(1-\mu_{A}\left(u_{i}\right)\right)^{2}\right)$, $C(A, B)=\Sigma_{i=1}^{n}\left\{\mu_{A}\left(u_{i}\right) \cdot \mu_{B}\left(u_{i}\right)+\left(1-\mu_{A}\left(u_{i}\right)\right) \cdot\left(1-\mu_{B}\left(u_{i}\right)\right)\right\}$.

11. $\operatorname{sim} 11=\min \left\{\alpha(A, B), \alpha\left(A^{c}, B^{c}\right)\right\}[6]$, where $\alpha(A, B)=\sqrt{\frac{\Sigma_{u \in U} \mu_{A}(u) \cdot \mu_{B}(u)}{\Sigma_{u \in U}\left(\max \left(\mu_{A}(u), \mu_{B}(u)\right)\right)^{2}}}$.

12. $\operatorname{sim} 12=\frac{1}{2}\left[(A \leftrightarrow B)+\left(A^{\prime} \leftrightarrow B^{\prime}\right)\right][10]$, where $A \leftrightarrow B=(A \rightarrow B) \wedge(B \rightarrow A), A^{\prime}=1-A$. $\wedge$ being a conjunction operator and $\rightarrow$ an implication operator. The above formula is equivalent to 
$S(A, B)=\frac{1}{n} \Sigma_{u \in U} \frac{1}{2}\left[\left(\mu_{A}(u) \leftrightarrow \mu_{B}(u)\right)+\left(\mu_{A^{\prime}}(u) \leftrightarrow \mu_{B^{\prime}}(u)\right)\right]$, where

$\left(\mu_{A}(u) \leftrightarrow \mu_{B}(u)\right)=\left(\mu_{A}(u) \rightarrow \mu_{B}(u)\right) \wedge\left(\mu_{B}(u) \rightarrow \mu_{A}(u)\right), n$ being the

cardinality of the universal set $U$, and $\wedge$ and $\rightarrow$ are defined logically. Now, if we

interpret the $\leftrightarrow$ implication operator as $\alpha \leftrightarrow \beta=1-|\alpha-\beta|$, then

$S(A, B)=1-\frac{1}{n} \Sigma_{i=1}^{n}\left|\mu_{A}(u)-\mu_{B}(u)\right|$.

13. $\operatorname{sim} 13=\frac{\operatorname{card}\left(\mu_{A}(u) \wedge \mu_{B}(u)\right)}{\max (\operatorname{card}(A), \operatorname{card}(B)))}$

14. $\operatorname{sim} 14=\frac{\operatorname{card}\left(\mu_{A}(u) \wedge \mu_{B}(u)\right)}{\operatorname{card}(A))}$

15. $\operatorname{sim} 15=\frac{\operatorname{card}\left(\mu_{A}(u) \wedge \mu_{B}(u)\right)}{\operatorname{card}(B))}$

16. $\operatorname{sim} 16=\min \left\{\frac{\operatorname{card}(A \cap B)}{\operatorname{card}(A)}, \frac{\operatorname{card}(A \cap B)}{\operatorname{card}(B)}\right\}$

A good working measure of similarity between two countable infinite fuzzy sets can be given as in the following:

$$
S(A, B)=1-\sup _{u \in U}\left|\mu_{A}(u)-\mu_{B}(u)\right| .
$$

It is easy to see that this can be an effective measure if we consider an infinite fuzzy set. But the problem in working with such a measure is that it gives importance to the sup operation only. The work in this regard can be found in [11].

Now, once a similarity index is defined - How can we compare this with other existing indices? How should we judge the goodness of such an index?

In this regard, the authors in [12,13] have reviewed different similarity measures, as suggested in the literature in the general case and as adapted to fuzzy sets. They have also presented an experimental design for linguistic approximation and discussed at length the suitability of application of different measures of similarity [14].

In [8], the authors presented a comparative study on the basis of a set of axioms. They have also investigated some similarities and dissimilarities in performance.

All the similarity measures listed above satisfy the reflexivity, symmetry and boundedness property. These three properties are indispensable for any similarity measures [9]. In this regard, all measures are equally useful. Besides these three properties, similarity measures should also satisfy properties like computational simplicity, monotonicity and non-dissimilarity. These are some desirable properties. Similarity measures based on the computation of overall sup as well as max between elements are such that they give more importance to a particular value and ignore the presence of others. Thus, two fuzzy sets are often found to be similar when they have the same sup and/or max. Of course, one can define two fuzzy sets to be similar as and when they have the same cardinality or they have the same support. This may work for mathematical theory construction. But in order to assist the decision maker in a real-life situation, the practical meaning of similarity concept is of vital importance. We are considering those indices that play a crucial role in the theory of fuzzy reasoning. This demands similarity measures based on separate membership degrees of each concept.

Next, let us consider the similarity measure defined in (4). In order to illustrate the drawback underlying it, let us consider a simple case as in the following:

$$
\begin{aligned}
\mu_{A}(u) & =1 \forall u \in U \text { and } \\
\mu_{B}(u) & =0 \text { for a particular } u_{0} \in U \text { and } \\
& =1 \text { otherwise. }
\end{aligned}
$$


Even in such an almost similar pair of fuzzy sets, it is found that the similarity index is 0 , showing thereby that they are completely dissimilar. Now, it can safely be concluded that it is practically impossible to single out one possible similarity measure that works well for all purpose.

In the following, we present an axiomatic definition of similarity between fuzzy sets, defined over the same universe of discourse. Some important deductions are also provided to illustrate the proposed measure's soundness. In order to provide a definition for similarity index, a number of factors must be considered. A primary consideration is that whatever way we choose to define such an index, it must satisfy the properties as already mentioned. Similarity measures are, in general, found to be non-transitive.

Under these circumstances, a similarity measure $S(A, B)$ should satisfy the following properties:

For all fuzzy sets $A, B$ :

P1. $S(B, A)=S(A, B)$.

P2. $S\left(A^{c}, B^{c}\right)=S(A, B), A^{c}$ being some negation of $A$.

P3. $0 \leq S(A, B) \leq 1$.

P4. $A=B$ if and only if $S(A, B)=1$.

P5. If $S(A, B)=0$, then either $A \cap B=\Phi$ (null) or $A^{c} \cap B^{c}=\Phi$, or $B=1-A$.

For $0 \leq \epsilon \leq 1$, if $S(A, B) \geq \epsilon$, we say that the two fuzzy sets $A$ and $B$ are $\epsilon$-similar. Thus, the case for $\epsilon=1$ correspond to equality of fuzzy sets. There could be many functions satisfying properties P1 through P5. One such measure of similarity satisfying properties P1 through P5 is given next.

Definition 4. Let $A=\sum_{u \in U} \mu_{A}(u) / u$ and $B=\sum_{u \in U} \mu_{B}(u) / u$ be two fuzzy sets defined over the same universe of discourse $U$. The similarity index of the pair $\{A, B\}$ is denoted by $S(A, B)$ and is defined by

$$
S(A, B)=1-\left(\frac{\sum_{u}\left|\mu_{A}(u)-\mu_{B}(u)\right|^{q}}{n}\right)^{\frac{1}{q}}
$$

where $n$ is the cardinality of the universe of discourse and $q \geq 1$ is the family parameter.

Theorem 4. If $S(A, B)=1$ and $S(B, C)=1$, then $S(A, C)=1$.

Theorem 5. For all fuzzy sets $A, B, C$, if either $A \subseteq B \subseteq C$ or $A \supseteq B \supseteq C$, then $S(A, C) \leq \min \{S(A, B), S(B, C)\}$.

Theorem 5 motivates us to consider the property of monotonicity of similarity between fuzzy sets to satisfy another axiom for some kind of monotonicity. So, we are now in a position to rewrite the axioms for similarity measure as in the following.

For all fuzzy sets $A, B$ and $C$ defined over the universe of discourse $U$, we have:

A1. $S(B, A)=S(A, B)$.

A2. $S\left(A^{c}, B^{c}\right)=S(A, B), A^{c}$ being some negation of $A$.

A3. $0 \leq S(A, B) \leq 1$. 
A4. $A=B$ if and only if $S(A, B)=1$.

A5. $S(A, B)=0$ if and only if $A \cap B=\Phi$.

A6. If $A \supseteq B \supseteq C$, then $S(A, B) \geq S(A, C)$.

Here, we note that $A^{c}$, the complement of a fuzzy set $A$, is to be defined first. We used the idea of '1-' as the complementation. On the basis of the above axioms, it is easy to see that the family of similarity measures defined in Definition 4 is a valid choice. All the measures satisfy axioms A1, A3, A4 and A5 for either identical or non-overlapping fuzzy sets.

Thus, we find that the similarity between fuzzy sets can be captured by aggregating the distinguishability between membership values of each element in the corresponding fuzzy sets. The similarity index between two fuzzy sets is a pure number and does not give any information about the inclusion. This explains why these measures are not transitive, in general. Let us tabulate the performance of different measures in the following.

\section{Performance of different similarity measures}

In this sub-section, we observe six cases (Figures 3, 4, 5, 6, 7 and 8), where each case compares two fuzzy sets in consideration. Also, for each case, 16 similarity measures mentioned earlier are calculated. A performance chart of various similarity measures are given in Table 1.

\section{Approximate reasoning}

Approximate reasoning is defined as the process or processes by which an approximate conclusion can be deduced from a set of possibly imprecise information using some inexact rule for the derivation. Since its inception in 1973, significant theoretical advances have established approximate reasoning as an important field of research. Different techniques of approximate reasoning have been proposed and discussed in the literature.

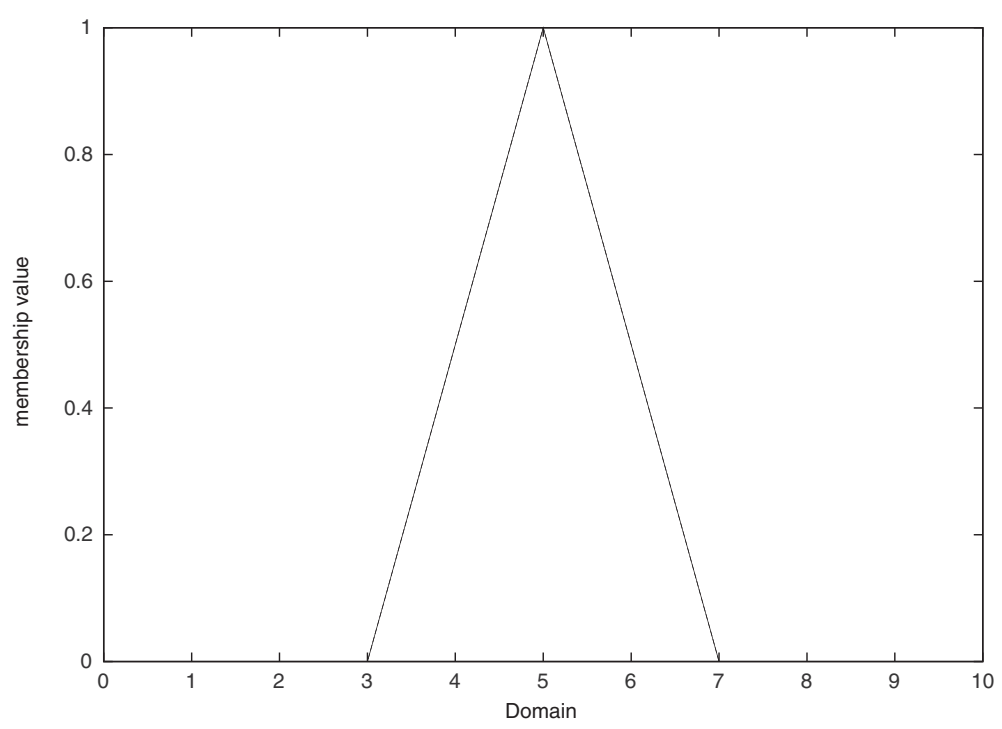

Figure 3 Case 1: two identical fuzzy sets. Fuzzy sets for measuring similarity. 


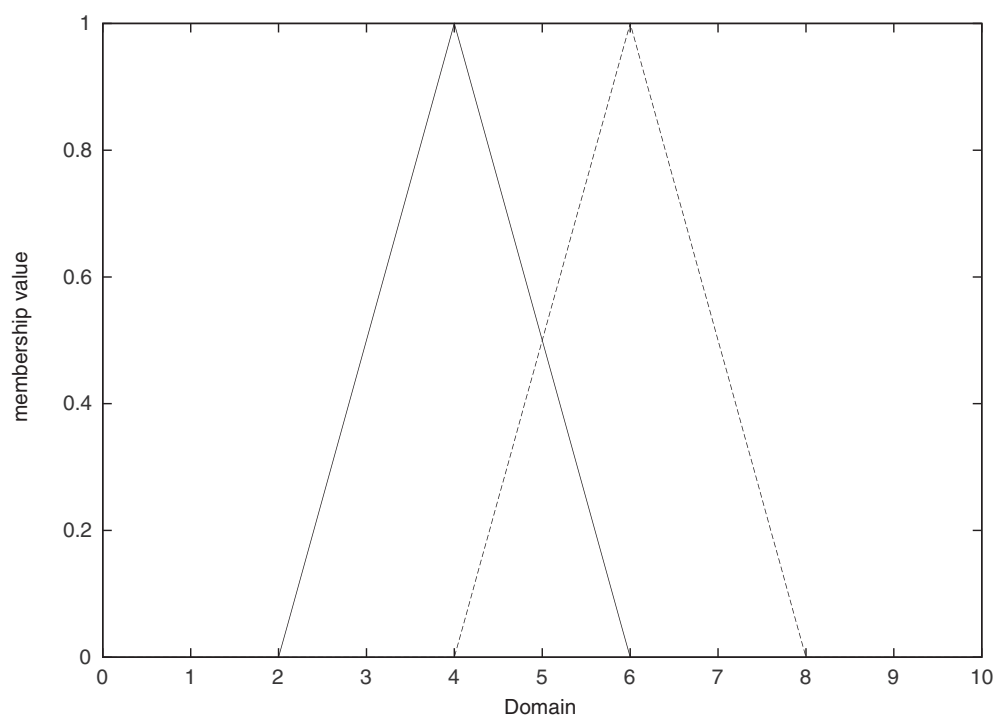

Figure 4 Case 2: two fuzzy sets almost close to each other. Fuzzy sets for measuring similarity.

Zadeh's [15] concept of approximate reasoning is based on fuzzy logic and the theory of fuzzy sets. In order to have an adequate understanding of the theory of approximate reasoning in this paper, some basic concepts are considered. The concept of linguistic variable plays an essential role in the theory of approximate reasoning. It is a tool for approximate characterisation of the values of the variables and their interrelations. For example, the height of a person may be short, the volume of a container may be huge, the code section of some programme may be tiny, two numbers may be approximately equal and so on. Zadeh [16] called such variables - linguistic variables.

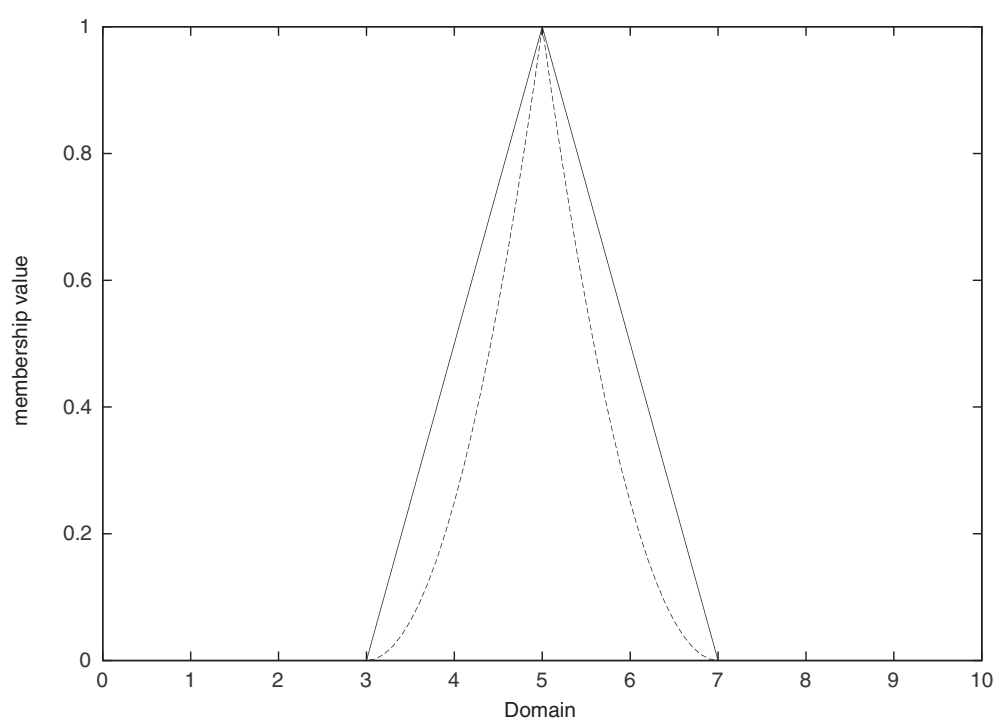

Figure 5 Case 3: two fairly close fuzzy sets. Fuzzy sets for measuring similarity. 


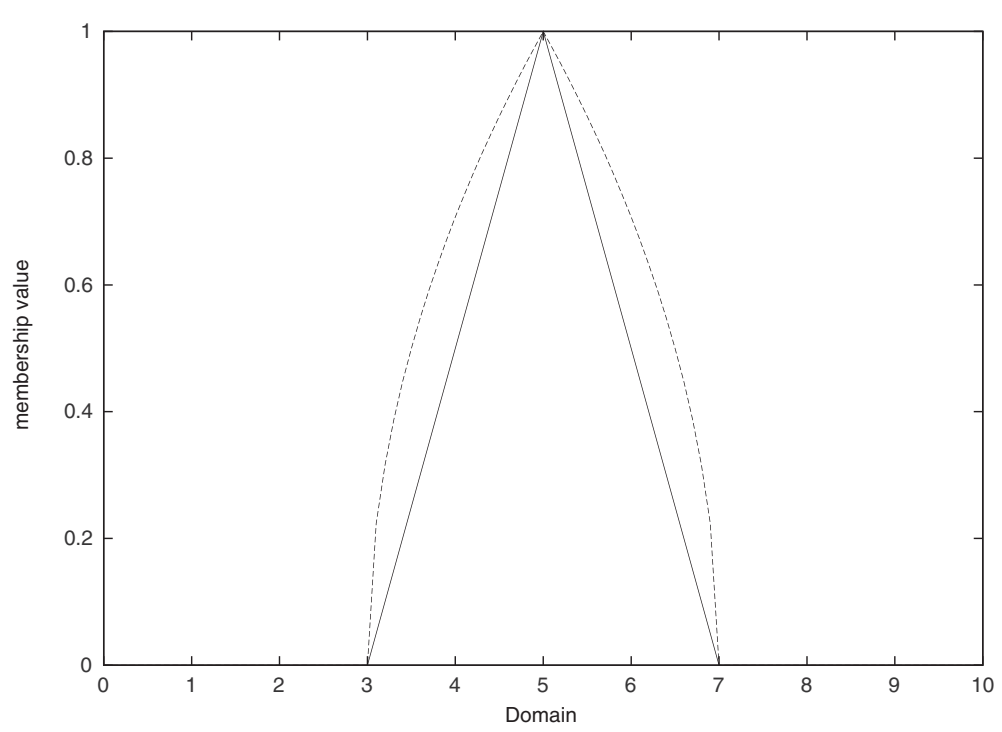

Figure 6 Case 4: two overlapping fuzzy sets. Fuzzy sets for measuring similarity.

The two basic inference rules considered for approximate reasoning based on fuzzy sets and fuzzy relations are the compositional rule of inference and the generalised modus ponens.

1. max-min composition. From ' $X$ is $A$ ' and ' $(X, Y)$ is $R$ ' infer ' $Y$ is $B$ ', where $\mu_{B}(y)=\max _{x} \min \left(\mu_{A}(x), \mu_{R}(x, y)\right)$.

2. Generalised modus ponens. From ' $X$ is $A^{*}$ ' and 'if $X$ is $A$, then $Y$ is $B^{\prime}$ infer ' $Y$ is $B^{*}$,' where $\mu_{B^{*}}(y)=\sup _{x}\left(\mu_{A^{*}}(x) \circ\left(\mu_{A}(x) \rightarrow \mu_{B}(y)\right)\right)$, o and $\rightarrow$ may have different interpretation.

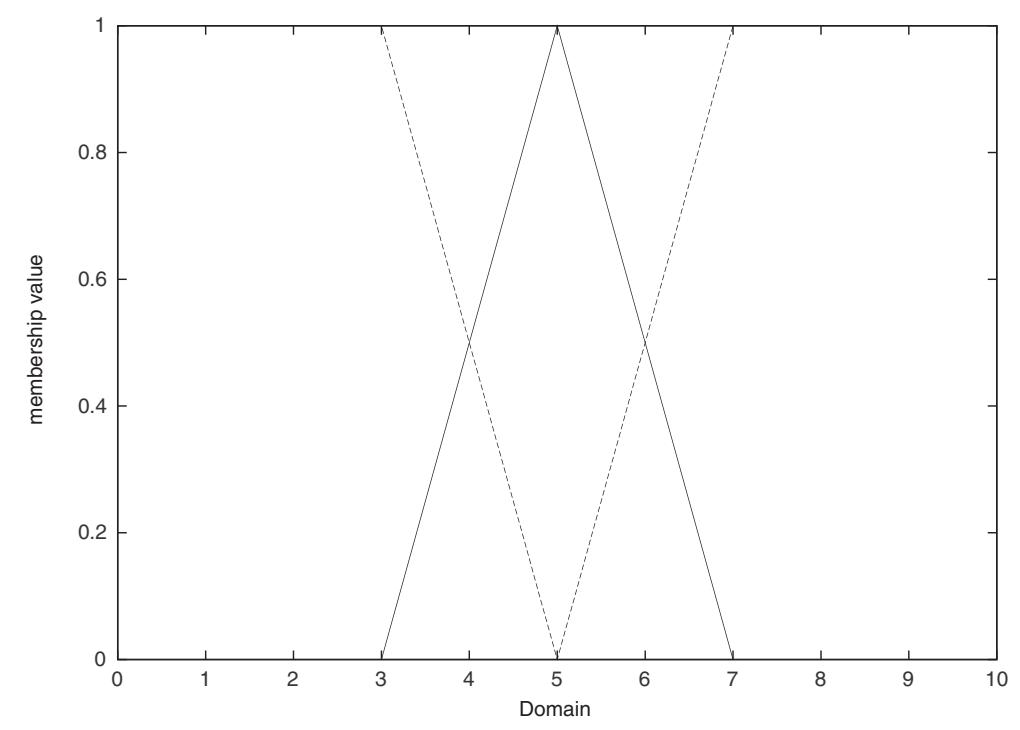

Figure 7 Case 5: two complementary fuzzy sets. Fuzzy sets for measuring similarity. 


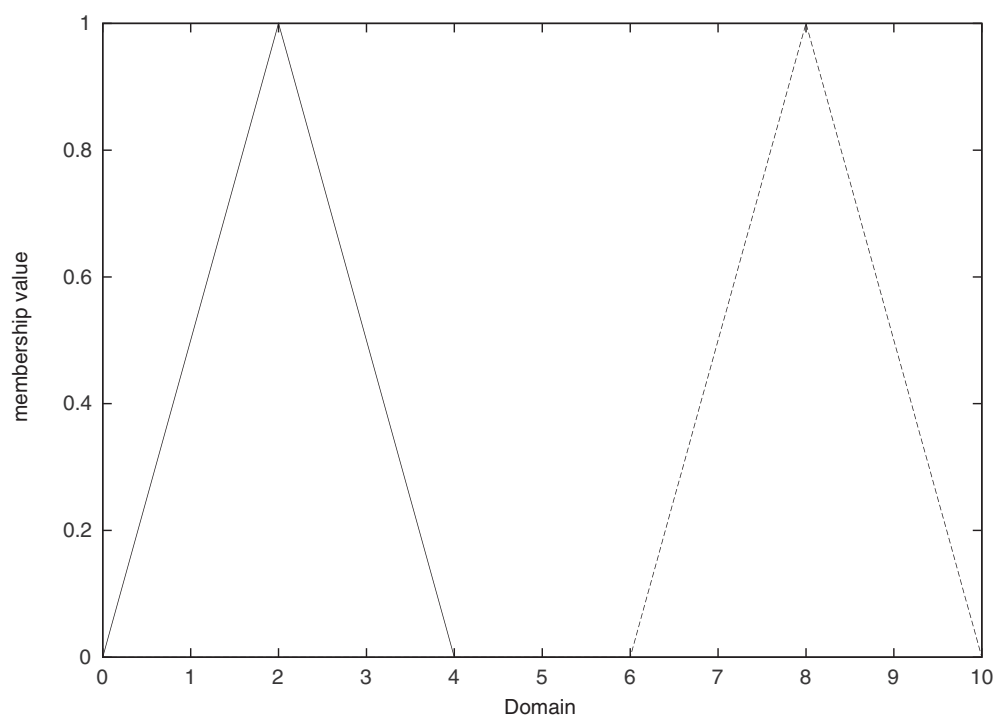

Figure 8 Case 6: two non-overlapping fuzzy sets. Fuzzy sets for measuring similarity.

Many fuzzy systems are based on Zadeh's compositional rule of inference [17]. Despite their success in various systems, researchers have indicated certain drawbacks [18] in the technique.

As for example, let $U=\left\{u_{1}, u_{2}, u_{3}, u_{4}\right\}$ and $V=\left\{v_{1}, v_{2}, v_{3}, v_{4}\right\}$ be the universes of discourse, $A=1.0 / u_{1}+0.75 / u_{2}+0.5 / u_{3}+0.25 / u_{4}$ and

$$
R=\begin{array}{c|cccc} 
& u_{1} & u_{2} & u_{3} & u_{4} \\
\hline v_{1} & 1.00 & 0.75 & 0.50 & 0.25 \\
v_{2} & 0.75 & 1.00 & 0.75 & 0.50 \\
v_{3} & 0.50 & 0.75 & 1.00 & 0.75 \\
v_{4} & 0.25 & 0.50 & 0.75 & 1.00
\end{array}
$$

\begin{tabular}{|c|c|c|c|c|c|c|c|c|}
\hline & \multicolumn{8}{|c|}{ Similarity measures } \\
\hline & $\operatorname{sim} 1$ & $\operatorname{sim} 2$ & $\operatorname{sim} 3$ & $\operatorname{sim} 4$ & $\operatorname{sim} 5$ & $\operatorname{sim} 6$ & $\operatorname{sim} 7$ & $\operatorname{sim} 8$ \\
\hline Case 1 & 1.00 & 1.00 & 1.00 & 1.00 & 1.00 & 1.00 & 1.00 & 1.00 \\
\hline Case 2 & 0.82 & 0.75 & 0.80 & 0.83 & 1.00 & 0.75 & 0.67 & 0.51 \\
\hline Case 3 & 0.82 & 0.75 & 0.86 & 0.83 & 1.00 & 0.80 & 0.75 & 0.68 \\
\hline Case 4 & 0.78 & 0.75 & 0.76 & 0.79 & 0.85 & 0.92 & 0.62 & 0.46 \\
\hline Case 5 & 0.14 & 0.00 & 0.20 & 0.20 & 0.50 & 0.09 & 0.11 & 0.15 \\
\hline \multirow[t]{2}{*}{ Case 6} & 0.41 & 0.00 & 0.00 & 0.48 & 0.00 & 0.00 & 0.00 & 0.00 \\
\hline & $\operatorname{sim} 9$ & $\operatorname{sim} 10$ & $\operatorname{sim} 11$ & $\operatorname{sim} 12$ & $\operatorname{sim} 13$ & $\operatorname{sim} 14$ & $\operatorname{sim} 15$ & $\operatorname{sim} 16$ \\
\hline Case 1 & 1.00 & 1.00 & 1.00 & 1.00 & 1.00 & 1.00 & 1.00 & 1.00 \\
\hline Case 2 & 1.00 & 0.79 & 0.87 & 0.87 & 0.67 & 0.67 & 1.00 & 0.67 \\
\hline Case 3 & 1.00 & 0.93 & 0.89 & 0.89 & 0.75 & 1.00 & 0.75 & 0.75 \\
\hline Case 4 & 0.46 & 0.85 & 0.79 & 0.83 & 0.76 & 0.76 & 0.76 & 0.76 \\
\hline Case 5 & 0.15 & 0.50 & 1.00 & 0.28 & 0.12 & 0.50 & 0.12 & 0.12 \\
\hline Case 6 & 0.00 & 0.00 & 0.00 & 0.00 & 0.00 & 0.00 & 0.00 & 0.00 \\
\hline
\end{tabular}

Then, taking $T=\min$ and using compositional rule of inference (CRI), we find $B=1.00 / v_{1}, 0.75 / v_{2}, 0.75 / v_{3}+0.50 / v_{4}$. This shows that the linguistic variables $X$

Table 1 Performance chart of various similarity measures 
and $Y$ are approximately equal. A careful scrutiny of the relation also says so. The conclusion $B$ will remain the same if we choose $A=1.0 / u_{1}+0.75 / u_{3}+0.50 / u_{4}$, which is highly dissimilar to $A$. Next, if we take $A=1.0 / u_{1}$, then from $R$ we have $B=1.00 / v_{1}+0.75 / v_{2}+0.50 / \nu_{3}+0.25 / v_{4}$; again, if we take $A=1.0 / u_{4}$, then $B=$ $0.25 / u_{1}+0.50 / u_{2}+0.75 / u_{3}+1.00 / u_{4}$. This shows that even if the input values are strongly complementary to each other, significant conclusions can be drawn using Zadeh's CRI.

This motivates the introduction of similarity-based reasoning techniques as proposed in [18-23].

\section{Similarity-based approximate reasoning}

To begin with, in this section, we will look at the different methods of inference based on a similarity measure. In [18,24-26], the authors proposed a similaritybased method called 'approximate analogical reasoning schema'. The method is applicable to both point-valued and interval-valued fuzzy sets. In [19], the author proposed two similar methods for medical diagnosis problems. Two other methods based on different modification procedures have been proposed in [27]. In the framework of existing approaches to similarity-based inference methodology, recently, in [21], the authors proposed two other similarity-based methods for reasoning and made a comparative study of the above similarity-based fuzzy reasoning methods.

In all these studies, it is proposed that similarity-based fuzzy reasoning methods do not require the construction of a fuzzy relation. Accordingly, they are based on the computation of the degree of similarity between the fact and the antecedent of a rule in a rule-based system. Then, based on the similarity value between the membership values of the elements of the fuzzy set representation of the fact and the corresponding fuzzy set in the antecedent of the rule, the membership value of each element of the consequent fuzzy set of the rule is modified to obtain a conclusion. This is the same for all existing similarity-based reasoning schemes. The modification procedure is different for different schemes.

We proposed two similarity-based approximate reasoning methods. One such method is a modification of the method presented in [18] and the other is a modification of Zadeh's compositional rule of inference. In the proposed methods, for inference in a rule-based system, the conditional rule is first expressed as a fuzzy binary relation. In translation, we prefer to use triangular norms for a better understanding. New facts are then used to compute the similarity between the fact and the antecedent of the rule to modify the above fuzzy binary relation and not the consequence of the rule as applied in the existing similarity-based reasoning techniques. The modification is based on a measure of similarity following some scheme to be presented. The result can be interpreted as the induced fuzzy binary relation. The inference is computed from the induced fuzzy binary relation using the well-known sup operation.

The above scheme is used in formulating different models (rule-based and resolutionbased). We will provide simple numerical examples for a better understanding of the proposed schemes.

In similarity-based approximate reasoning scheme, we see that from a given fact, the desired conclusion is derived using only a measure of similarity between the fact and 
the antecedent in a rule-based system. In some cases, a threshold value $\tau$ is associated with a rule. If the degree of similarity, between the antecedent of the rule and the given fact, exceeds the real value of $\tau$, associated with the rule under consideration, only then is that rule assumed to be fired. The conclusion is derived using some modification procedure.

As an illustration, let us consider the two premises as in Table 2.

Here, $A$ and $A^{\prime}$ are fuzzy sets defined over the same universe of discourse $U=$ $\left\{u_{1}, u_{2}, \ldots, u_{m}\right\}$ and $B, B^{\prime}$ are defined over the universe of discourse $V=\left\{v_{1}, v_{2}, \ldots, v_{n}\right\}$. Let $S\left(A, A^{\prime}\right)$ denote some measure of similarity between two fuzzy sets $A$ and $A^{\prime}$. In the existing techniques, if $S\left(A, A^{\prime}\right)>\tau$, then the rule will be fired and the consequent of the rule is modified to produce the desired conclusion. Based on the change of membership grade of the consequent, two types of modification procedures can be proposed as in $[18,28]$ - expansion-type inference and reduction-type inference.

Let $B^{\prime}=\sum_{i=1}^{n}\left\{\mu_{B^{\prime}}\left(v_{i}\right) / v_{i}\right\}$ and $s=s(S(A, B), \tau)$.

Expansion form: $\mu_{B^{\prime}}\left(v_{i}\right)=\min \left(1, \mu_{B}\left(v_{i}\right) / s\right)$. Reduction form: $\mu_{B^{\prime}}\left(v_{i}\right)=\left(\mu_{B}\left(v_{i}\right) . s\right)$.

The methods proposed in $[19,29]$ use the threshold value, a confidence factor and the reduction form of inference without providing any argument as to the choice of modification procedure. In one of them [19], each fuzzy set is first conceived as an $m$-component vector, and then the concept of vector dot product is used for finding the similarity. If $S\left(A, A^{\prime}\right) \geq \tau$, the predefined threshold value, then the rule will be fired and strength of confirmation is calculated by $S\left(A, A^{\prime}\right) . \mu$, where $\mu$ is the membership value associated with the rule. In the other method [29], the author used weights with each propositions for the calculation of similarity. The procedure for the computation of the conclusion remains the same.

In [27], the authors used the value of certainty factor associated with the rules in the modification procedure. The inference is based on the number of propositions in the antecedent of the rule(s) as also the operator(s) connecting them. In each case, the inference is one of expansion type. In [21], they have also presented two more modification procedures and claimed for two new fuzzy reasoning methods. One modification is based on Zadeh's inclusion and cardinality measure and the other on equality and cardinality measure. Other operations remain almost identical.

\section{Proposed method}

In this section, we show how conclusions can be obtained from the given premises with the help of such a similarity measure. Let $X, Y$ be two linguistic variables and let $U, V$ respectively denote the universes of discourse. Two typical propositions $p$ and $q$ are given, and we like to derive a conclusion according to similarity-based inference. The scheme can be best described in Table 2 .

Table 2 Ordinary approximate reasoning

\begin{tabular}{llll}
\hline$p:$ & $X$ is $A$ & then & $Y$ is $B$ \\
$q:$ & $X$ is $A^{\prime}$ & \\
\hline$r:$ & & $Y$ is $B^{\prime}$. \\
\hline
\end{tabular}


Let $U=\left\{u_{1}, u_{2}, \ldots, u_{l}\right\}, \quad V=\left\{v_{1}, v_{2}, \ldots, v_{m}\right\}$ denote the respective universe of discourse of the linguistic variables $X$ and $Y$. Let fuzzy sets $A, A^{\prime}$ and $B$ in Table 2 be defined as

$$
\begin{aligned}
& A=\sum_{i=1}^{l}\left\{\mu_{A}\left(u_{i}\right) / u_{i}\right\} ; A^{\prime}=\sum_{i=1}^{l}\left\{\mu_{A^{\prime}}\left(u_{i}\right) / u_{i}\right\} ; \\
& B=\sum_{i=1}^{m}\left\{\mu_{B}\left(v_{i}\right) / v_{i}\right\} ; B^{\prime}=\sum_{i=1}^{m}\left\{\mu_{B^{\prime}}\left(v_{i}\right) / v_{i}\right\} .
\end{aligned}
$$

All the existing methods $[18,20,21,27]$ use the similarity measure for a direct computation of inference without considering the induced relation, i.e. how the underlying relation (a condition) is modified in the presence of the given fact. This is important in deriving a consequence of the fact from the rule. Consequently, those methods provide the same conclusion, if $A$ and $A^{\prime}$ are interchanged in the propositions concerned. Thus, if $p, q$ and $p^{\prime}, q^{\prime}$ be defined as in the following:

(i) $p:$ if $X$ is $A$, then $Y$ is $B, \tau$ and $q: X$ is $A^{\prime}$;

(ii) $p:$ if $X$ is $A^{\prime}$, then $Y$ is $B, \tau$ and $q: X$ is $A$,

then both $(i)$ and (ii) will produce the same conclusion which is not appealing. This happens because the conclusion is derived by a modification of the consequent of the rule. It should be noted here that this is not the case with Zadeh's compositional rule of inference. Another notable fact is that we need to consider the threshold or certainty factor in order to tackle the problem of rule misfiring.

The first drawback can be eliminated if we consider the interpretation of the relational operator present in the conditional premise, as is done in executing compositional rule of inference. It is easy to verify that for a class of nested fuzzy sets, each different from the other, the consequence of a rule using CRI becomes the same. We seek a reasoning system which should be such that every change in the concept(s) as appears in the antecedent of the rule and that in the fact should be incorporated in the induced relation between the variables defining a rule, in this case, $X$ and $Y$. Only then the inference will be influenced by the change concerned.

In order to avoid the use of certainty factor for rule misfiring, we modify the inference scheme in such a way that a significant change will make the conclusion less specific. This is done if an expansion type of inference scheme be chosen. Here, the 'UNKNOWN' case, i.e. the fuzzy set $B^{\prime}=V$, can be taken as the limit. Explicitly, when the similarity value becomes low, i.e when $A$ and $A^{\prime}$ differ significantly, the reasoning process should be such that the only inference be $B^{\prime}=V$. As $A^{\prime}=A$, we expect that $B^{\prime}=B$. This, in turn, implies that nothing better than what the rule says should be allowed as a valid conclusion.

\section{Schema}

In view of the above observations, we propose a similarity-based inference method for deriving the consequence $r$. We first generate the fuzzy relation between the antecedent variable(s) and the consequent variable as done in executing CRI. We then compute the absolute change in linguistic labels, represented as fuzzy sets, and systematically propagate the same into the conditional relation in order to obtain the induced modified conditional relation. From this induced modified relation, a possible conclusion can be 
drawn using the sup operation. The scheme for computation can be presented in the following algorithm.

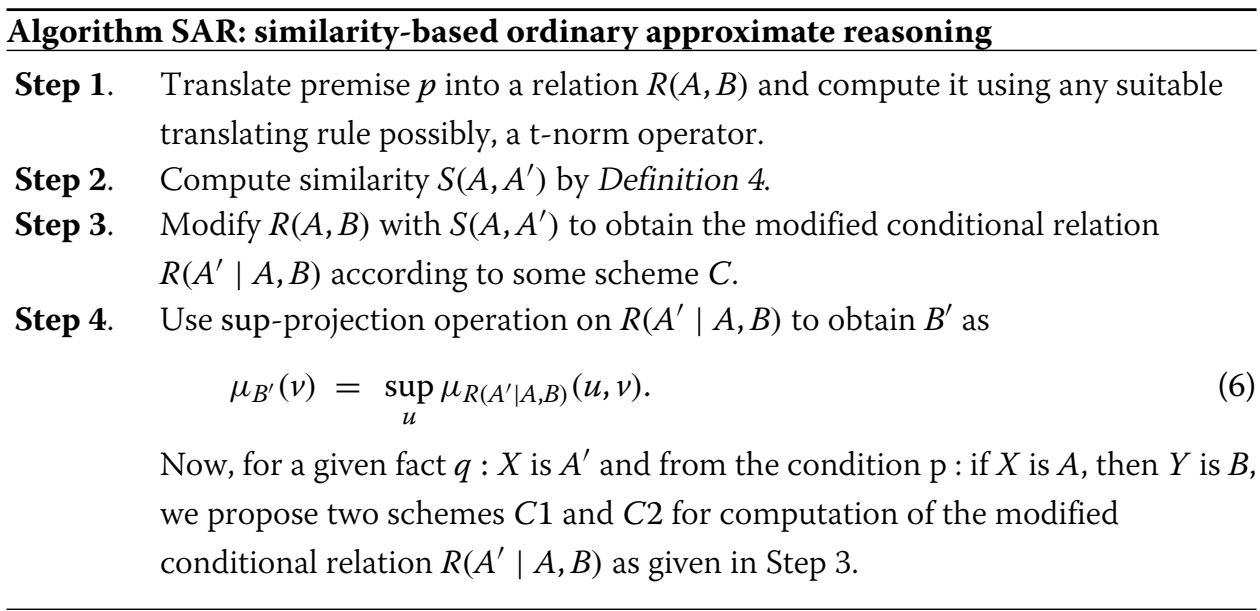

Scheme $\mathrm{C1}$. The first scheme $\mathrm{C} 1$ is based on a concept similar (but NOT identical) to the method proposed in [18]. We may recall here that the authors computed the conclusion $B^{\prime}=\min (1, B / s)$, where $s$ is the measure of similarity between fuzzy sets $A$ and $A^{\prime}$ without considering the information suggested by the conditional rule. Here, we propose to modify the conditional relation according to (7).

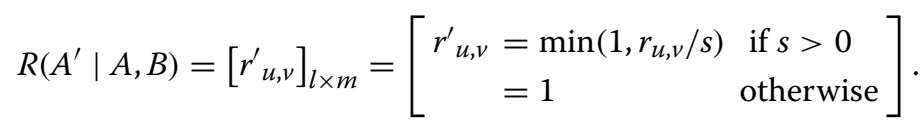

The difference between the proposed scheme from the one presented in [18] can be noted easily. It is clear that the proposed scheme, unlike the schemes in $[18,20]$, does not produce the same conclusion when $A$ and $A^{\prime}$ are interchanged. It is not difficult to see that in (7), if $s \leq r_{u, v}$ for some $v \in V$, then $r^{\prime}{ }_{u, v}$ becomes equal to 1 . This makes the membership of that $v$ in the resultant fuzzy set equal to one. This scheme, although a heuristic one, is intuitively a plausible scheme. Our next scheme $\mathrm{C} 2$ for computation of $R\left(A^{\prime} \mid A, B\right)$ is based on a set of axioms.

Scheme $C 2$. We believe that in a similarity-based reasoning methodology, a scheme for computation of the induced relation, when a fact and a conditional statement is given, should satisfy the following axioms:

A1. If $S\left(A, A^{\prime}\right)=1$, i.e. if $A^{\prime}=A$, then $\mu_{R\left(A^{\prime} \mid A, B\right)}(u, v)=\mu_{R(A, B)}(u, v), \forall(u, v) \in U \times V$.

A2. If $S\left(A, A^{\prime}\right)=0$, i.e. if $A^{\prime} \cap A=\Phi$, then $\mu_{R\left(A^{\prime} \mid A, B\right)}=1 \quad \forall(u, v) \in U \times V$.

A3. As $S\left(A, A^{\prime}\right)$ increase from 0 to $1, \mu_{R\left(A^{\prime} \mid A, B\right)}(u, v)$ decreases uniformly from 1 to

$$
\mu_{R(A, B)}(u, v) ; \forall(u, v) \in U \times V .
$$

Axiom $\mathrm{A} 1$ asserts that we should not modify the conditional relation as and when $A^{\prime}$ and $A$ remain equal. Axiom A2 asserts that when $A^{\prime}$ is completely dissimilar to $A$, i.e. $A^{\prime}$ and $A$ have disjoint support, we should not conclude specifically. In such a situation, anything is possible. A3 says that as the fact $A^{\prime}$ changes from the most dissimilar case (similarity value 0 ) to the most similar one (similarity value 1 ), the inferred conclusion should change from the most non-specific case, i.e. the UNKNOWN case $\left(B^{\prime}=V\right)$ to the most specific 
case, i.e. $B^{\prime}=B$. This, in turn, means that whatever $A^{\prime}$ be, $R\left(A^{\prime} \mid A, B\right) \supseteq R(A, B)$, i.e. the induced relation should not be more specific than what is given as a condition.

For notational simplicity, let us denote $S\left(A, A^{\prime}\right)$ by $s$ and $R_{A^{\prime} \mid A, B}$ by $r^{\prime}$. Now, axiom A3 uniquely suggest a function of the form

$$
\frac{d r^{\prime}}{d s}=k(\text { a constant }) \Rightarrow r^{\prime}=k s+c, c \text { is a constant. }
$$

These two constants can be determined from the conditions already prescribed in axiom A1 and axiom A2. More explicitly, when $s=1$, we know that $r^{\prime}=r$ (from axiom $\mathrm{A} 1$ ), and when $s=0$, we know that $r^{\prime}=1$ (from axiom A2). This gives, $r^{\prime}=1-(1-r) . s$ as our new scheme for the modification of the conditional relational.

Therefore, axiom A1 through axiom A3 uniquely suggest the scheme C2 as

$$
\mu_{R\left(A^{\prime} \mid A, B\right)}(u, v)=1-\left(1-\mu_{R(A, B)}(u, v)\right) . S\left(A, A^{\prime}\right) .
$$

From (7) and (8), we observe that when $S\left(A, A^{\prime}\right)=0$ we have $B^{\prime}=V$. In other words, it is impossible to conclude anything when $\left\{A, A^{\prime}\right\}$ are completely dissimilar. Again, when $S\left(A, A^{\prime}\right)$ is close to unity, then $R\left(A^{\prime} \mid A, B\right)$ is close to $R(A, B)$ and the inferred fuzzy set $B^{\prime}$ will be close to $B$, i.e. $S\left(B, B^{\prime}\right)$ is close to unity. Axiom A3 also suggests that a small change in the input produces a small change in the output. In this sense, the above mechanism of inference is stable. As in the previous case, in (8), if either $S\left(A, A^{\prime}\right)=0$ or $\mu_{R(A, B)}=1$, then $r_{u, v}^{\prime}$ becomes equal to 1 .

Let us see how the above scheme can be modified to handle the concept of threshold associated with a rule in a natural manner. Let $\tau$ be the threshold associated with the rule. For that, we are to modify axiom A2 according to the following:

A4. If $S\left(A, A^{\prime}\right) \leq \tau$, then $\mu_{R\left(A^{\prime} \mid A, B\right)}=1 \forall(u, v) \in U \times V$. Accordingly, simple calculations as before resulted in the following

$\mu_{R\left(A^{\prime} \mid A, B\right)}=\min \left[1,\left(1-\left(1-\mu_{R(A, B)}\right) \cdot \frac{s-\tau}{1-\tau}\right)\right]$ as the general scheme for relation membership modification. The case $\tau=0$ corresponds to the scheme presented in (8). This scheme ensures that with all fuzzy sets $A^{\prime}$ having similarity value $S\left(A, A^{\prime}\right)$ less or equal to the threshold value $\tau$, the inference $B^{\prime}$ using (7) will be 'UNKNOWN'.

Let $A$ be a normal fuzzy set. If we assume that the translating rule used in generating the conditional relation is one of the t-norm types, then, as is already proposed, a basic and desirable result of the inferred proposition nothing better than what the rule says can be concluded can be established as in the following. For that, let us consider the model as in Table 2. For all $A, A^{\prime}$, the following proposition is valid.

Theorem 6. $B^{\prime} \supseteq B . \quad[5,30]$

A few translation rules are presented in Table 3, and the corresponding rules for modification of relations are presented in Table 4 , based on the interpretation of the $\rightarrow$ operators in the formula $\left(s \rightarrow \mu_{R}\right.$ ), where $s$ is the similarity measure between $A$ and $A^{\prime}$, i.e. $s=S\left(A, A^{\prime}\right)$. They can be categorised into groups - expansion-type modifier and reduction-type modifier. For the first class, $\mu_{R^{\prime}} \geq \mu_{R} \forall(u, v) \in U \times V$, and that for the second class, $\mu_{R^{\prime}} \leq \mu_{R} \forall(u, v) \in U \times V$. 
Table 3 For translation of relations

\begin{tabular}{|c|c|c|}
\hline$R$ & Conditional relations & Translation $\left(\mu_{R}(u, v)\right)$ \\
\hline$\overline{R_{1}}$ & Reichenbach s-implication & $1-\mu_{A}(u)+\mu_{A}(u) * \mu_{B}(v)$ \\
\hline$R_{2}$ & Kleen-Dienes s-implication & $\max \left(1-\mu_{A}(u), \mu_{B}(v)\right)$ \\
\hline$R_{3}$ & Lukasiewicz r- and s-implications & $\min \left(1-\mu_{A}(u)+\mu_{B}(v), 1\right)$ \\
\hline$R_{4}$ & Rescher-Gaines r-implication & $\begin{cases}1, & \left.\text { if } \mu_{A}(u) \leq \mu_{B}(v)\right) \\
0, & \text { otherwise }\end{cases}$ \\
\hline$R_{5}$ & Godel r-implication & $\begin{cases}1, & \left.\text { if } \mu_{A}(u) \leq \mu_{B}(v)\right) \\
\mu_{B}(v), & \text { otherwise }\end{cases}$ \\
\hline$R_{6}$ & Goguen r-implication & $\begin{cases}\min \left(\mu_{B}(v) / \mu_{A}(u), 1\right), & \text { if } \mu_{A}(u)>0 \\
1, & \text { otherwise }\end{cases}$ \\
\hline$R_{7}$ & Standard t-norm & $\min \left(\mu_{A}(u), \mu_{B}(v)\right)$ \\
\hline$R_{8}$ & Bounded sum t-norm & $\max \left(\mu_{A}(u)+\mu_{B}(v)-1,0\right)$ \\
\hline$R_{9}$ & Product t-norm & $\mu_{A}(u) * \mu_{B}(v)$ \\
\hline
\end{tabular}

A few examples of translating rules for a simple conditional statement is presented in Table 3. Each rule actually identifies a fuzzy relation. With input, these relations are modified using schemes presented in Table 4 . The effects of different translating rules and modification procedures are presented in Table 5 , which shows that for identical matching as in Figure 9, the output of rule firing is consistent if we choose t-norm and r-implication for translation.

For distinct $A, A^{\prime}$ as in Figure 10, the result of rule firing is shown in Table 6. In this case, also, the performance of translating rules is satisfactory for t-norm $\left(R_{7}, R_{8}, R_{9}\right)$ as well as r-implication $\left(R_{4}, R_{5}, R_{6}\right)$, and modification rules $\mathrm{mr} 1, \mathrm{mr} 2$ and $\mathrm{mr} 3$, respectively. Simulation study reveals the modification procedure mr2 is consistent. An output fuzzy set $B^{\prime}$ is shown in Figure 11, using the modification scheme mr1 in Table 6.

With the above understanding of similarity-based reasoning methodology, let us now propose another module of a fuzzy system - defuzzification.

\section{Specificity measure - defuzzification}

The result of rule firing, using any of the above-mentioned approaches to inference, is a fuzzy set. This is interpreted at the semantic level as the desired output. Often, we need to determine a precise action as output. The purpose of defuzzification is to obtain a scalar value $u \in U$, from the said output fuzzy set, as the action. Then, if necessary, denormalisation is performed on the output so as to obtain the corresponding action on its physical domain.

Table 4 For modification of relations

\begin{tabular}{|c|c|c|}
\hline Scheme & Modified relations $\mu_{R^{\prime}}(u, v)$ & Type \\
\hline mr1 & $\begin{cases}\min \left(\mu_{R}(u, v) / s, 1\right), & \text { if } s>0 \\
1, & \text { otherwise }\end{cases}$ & Expansion \\
\hline $\mathrm{mr} 2$ & $\min \left((1-s)+s \cdot \mu_{R}(u, v), 1\right)$ & Reduction \\
\hline $\mathrm{mr3}$ & $\min \left(1-s+\mu_{R}(u, v), 1\right)$ & Expansion \\
\hline mr4 & $\max \left(1-s \cdot \mu_{A}(u), \mu_{B}(v)\right)$ & Reduction \\
\hline $\mathrm{mr} 5$ & $\max \left(1-s \cdot \mu_{A}(u), \min \left(\mu_{A}(u), \mu_{B}(v)\right)\right)$ & \\
\hline mr6 & $\max \left(1-s, \min \left(s, \max \left(1-\mu_{A}(u), \min \left(\mu_{A}(u), \mu_{B}(v)\right)\right)\right)\right)$ & \\
\hline $\mathrm{mr} 7$ & $\min \left(1-\mu_{A}(u) \cdot s+s \cdot \mu_{A}(u) \cdot \mu_{B}(v), 1\right)$ & \\
\hline
\end{tabular}


Table 5 Results for different reasoning schemes for $\boldsymbol{A}^{\prime}=\boldsymbol{A}$

\begin{tabular}{lccccc}
\hline Modified relation & Relation data $\left(\boldsymbol{A}^{\prime}\right)$ & Input & $\boldsymbol{S}\left(\boldsymbol{A}, \boldsymbol{A}^{\prime}\right)$ & Output $\left(\boldsymbol{B}^{\prime}\right)$ & $\boldsymbol{S}\left(\boldsymbol{B}, \boldsymbol{B}^{\prime}\right)$ \\
\hline $\mathrm{mr} 1, \mathrm{mr} 2, \mathrm{mr} 3$ & $R_{1}$ & $A$ & 1.0 & $B^{\prime} \supset B$ & 0.524941 \\
& $R_{2}$ & $A$ & 1.0 & $B^{\prime} \supset B$ & 0.526477 \\
& $R_{3}$ & $A$ & 1.0 & $B^{\prime} \supset B$ & 0.522559 \\
& $R_{4}$ & $A$ & 1.0 & $B^{\prime}=B$ & 1.0 \\
& $R_{5}$ & $A$ & 1.0 & $B^{\prime}=B$ & 1.0 \\
& $R_{6}$ & $A$ & 1.0 & $B^{\prime} \supset B$ & 0.945352 \\
& $R_{7}$ & $A$ & 1.0 & $B^{\prime}=B$ & 1.0 \\
& $R_{8}$ & $A$ & 1.0 & $B^{\prime}=B$ & 1.0 \\
& $R_{9}$ & $A$ & 1.0 & $B^{\prime}=B$ & 1.0 \\
$\mathrm{mr} 4$ & $A$ & 1.0 & $B^{\prime} \supset B$ & 0.526477 \\
$\mathrm{mr} 5$ & & 1.0 & $B^{\prime} \supset B$ & 0.526477 \\
$\mathrm{mr} 6$ & & $A$ & $B^{\prime} \supset B$ & 0.526477 \\
$\mathrm{mr} 7$ & & $A$ & 1.0 & $B^{\prime} \supset B$ & 0.524941 \\
\hline
\end{tabular}

Specificity measure of fuzzy set estimates the precision of an information represented by the fuzzy set rather than an estimate of its fuzziness which is measured by the entropy of the fuzzy set. In order to provide a definition for any specificity index, a number of factors must be considered. A fuzzy set with maximum specificity value corresponds to a precise assessment of the values of a variable. In trying to capture the form of the specificity index, a number of properties are required or desirable.

According to Dubois and Prade, a specificity measure $\operatorname{Sp}(A)$ [31] should satisfy the following properties. Let $X$ be a linguistic variable defined on a universe of discourse $U$. $A$ and $B$ are normalised fuzzy subsets of $U$.

P1. $\forall A \subseteq U, \operatorname{Sp}(A) \in[0,1]$.

P2. $\operatorname{Sp}(A)=1$ if and only if $A$ is a singleton of $S$.

P3. If $A \subseteq B$, then $\mathrm{Sp}(A) \geq \operatorname{Sp}(B)$.

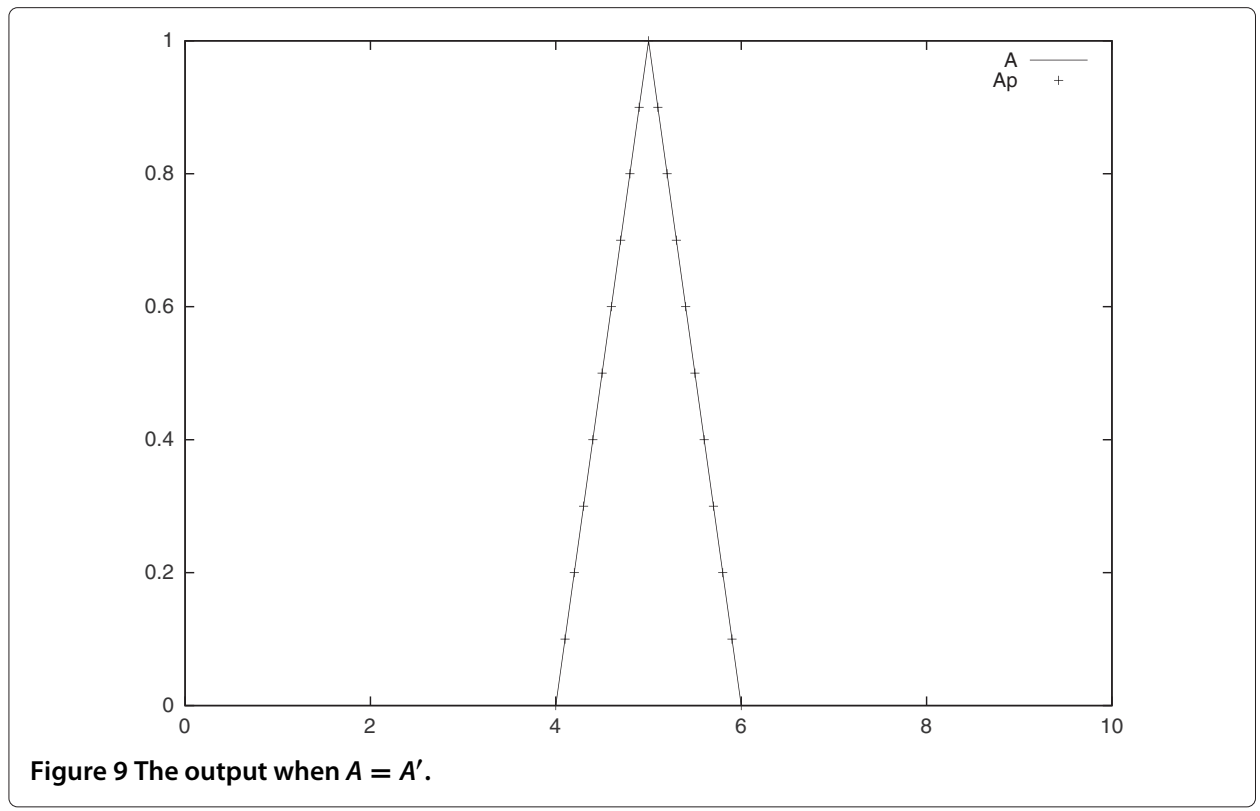




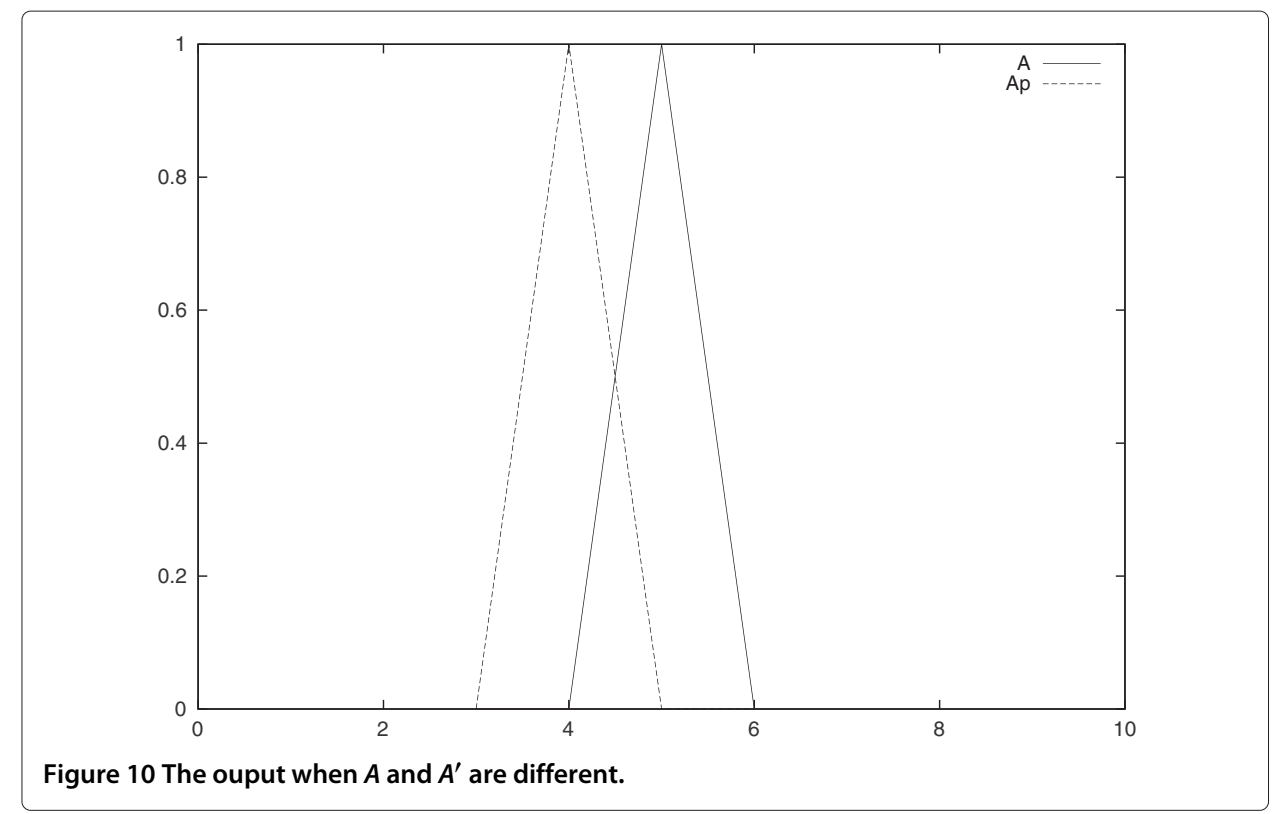

Yager [32] introduced one such measure of specificity that satisfies the above properties. When $U$ is finite, Yager proposed an expression for defining the specificity. Let us assume that $A$ be a fuzzy set defined over the universal set $U$ and $A_{\alpha}$ be the $\alpha$-level set of $A$. The specificity associated with $A$ is denoted as $\operatorname{Sp}(A)$ and is defined as

$$
\operatorname{Sp}(A)=\int_{0}^{\alpha_{\max }} \frac{1}{\operatorname{card} A_{\alpha}} d \alpha
$$

Let us now list some properties [32] associated with the above definition.

For all $A, \operatorname{Sp}(A)$ assumes its maximum value 1 , when $A=\{1 / u\}$ for some particular $u \in U$.

For all $A, \operatorname{Sp}(A) \in[0,1]$ and it assumes its minimum value 0 , when $A=\Phi$.

If, for all $A, \mu_{A}(u)=k$ for all $u \in U$, then $\operatorname{Sp}(A)=\frac{k}{n}$ where $n$ is the cardinality of the ordinary set $U$.

Defuzzification is a procedure applied to reduce the anxiety in a decision. Accordingly, we propose a new technique for defuzzification based on a measure of precision. Let there be $m$ clipped fuzzy sets $\left\{A^{(k)} ; k=1,2, \ldots, m\right\}$ and let $\left\{s_{(k)}, p^{(k)} ; k=1,2, \ldots, m\right\}$ be the specificity associated with $A^{(k)}$ as well as the peak of the consequent fuzzy set of the $k$ th rule. Then, the defuzzified value $u^{*}$ will be given by

$$
u^{*}=\frac{\sum_{k=1}^{m} p^{(k)} \cdot s_{(k)}}{\sum_{k=1}^{m} s_{(k)}}
$$

The height method of defuzzification demands strictly convex fuzzy sets. The individual peak values of consequent fuzzy sets of the fired rules are used to generate the weighted average of these peak values. It is a simple method and works faster than the centre of 
Table 6 Results for different reasoning schemes for $\boldsymbol{A}^{\prime} \neq \boldsymbol{A}$

\begin{tabular}{|c|c|c|c|c|c|}
\hline Modified relation & Relation & Input data $\left(A^{\prime}\right)$ & $S\left(A, A^{\prime}\right)$ & Output $\left(B^{\prime}\right)$ & $S\left(B, B^{\prime}\right)$ \\
\hline \multirow[t]{9}{*}{$\mathrm{mr1}$} & $R_{1}$ & $A^{\prime}$ & 0.683772 & $B^{\prime} \supset B$ & 0.438764 \\
\hline & $R_{2}$ & $A^{\prime}$ & 0.683772 & $B^{\prime} \supset B$ & 0.441981 \\
\hline & $R_{3}$ & $A^{\prime}$ & 0.683772 & $B^{\prime} \supset B$ & 0.437005 \\
\hline & $R_{4}$ & $A^{\prime}$ & 0.683772 & $B^{\prime}=B$ & 1.0 \\
\hline & $R_{5}$ & $A^{\prime}$ & 0.683772 & $B^{\prime} \supset B$ & 0.935526 \\
\hline & $R_{6}$ & $A^{\prime}$ & 0.683772 & $B^{\prime} \supset B$ & 0.906437 \\
\hline & $R_{7}$ & $A^{\prime}$ & 0.683772 & $B^{\prime} \supset B$ & 0.935526 \\
\hline & $R_{8}$ & $A^{\prime}$ & 0.683772 & $B^{\prime} \supset B$ & 0.935526 \\
\hline & $R_{9}$ & $A^{\prime}$ & 0.683772 & $B^{\prime} \supset B$ & 0.935526 \\
\hline \multirow[t]{9}{*}{$\mathrm{mr} 2$} & $R_{1}$ & $A^{\prime}$ & 0.683772 & $B^{\prime} \supset B$ & 0.437789 \\
\hline & $R_{2}$ & $A^{\prime}$ & 0.683772 & $B^{\prime} \supset B$ & 0.439834 \\
\hline & $R_{3}$ & $A^{\prime}$ & 0.683772 & $B^{\prime} \supset B$ & 0.436249 \\
\hline & $R_{4}$ & $A^{\prime}$ & 0.683772 & $B^{\prime} \supset B$ & 0.707154 \\
\hline & $R_{5}$ & $A^{\prime}$ & 0.683772 & $B^{\prime} \supset B$ & 0.697115 \\
\hline & $R_{6}$ & $A^{\prime}$ & 0.683772 & $B^{\prime} \supset B$ & 0.691508 \\
\hline & $R_{7}$ & $A^{\prime}$ & 0.683772 & $B^{\prime} \supset B$ & 0.697115 \\
\hline & $R_{8}$ & $A^{\prime}$ & 0.683772 & $B^{\prime} \supset B$ & 0.697115 \\
\hline & $R_{9}$ & $A^{\prime}$ & 0.683772 & $B^{\prime} \supset B$ & 0.697115 \\
\hline \multirow[t]{9}{*}{$\mathrm{mr} 3$} & $R_{1}$ & $A^{\prime}$ & 0.683772 & $B^{\prime} \supset B$ & 0.407110 \\
\hline & $R_{2}$ & $A^{\prime}$ & 0.683772 & $B^{\prime} \supset B$ & 0.410883 \\
\hline & $R_{3}$ & $A^{\prime}$ & 0.683772 & $B^{\prime} \supset B$ & 0.405919 \\
\hline & $R_{4}$ & $A^{\prime}$ & 0.683772 & $B^{\prime} \supset B$ & 0.701754 \\
\hline & $R_{5}$ & $A^{\prime}$ & 0.683772 & $B^{\prime} \supset B$ & 0.687924 \\
\hline & $R_{6}$ & $A^{\prime}$ & 0.683772 & $B^{\prime} \supset B$ & 0.682404 \\
\hline & $R_{7}$ & $A^{\prime}$ & 0.683772 & $B^{\prime} \supset B$ & 0.687924 \\
\hline & $R_{8}$ & $A^{\prime}$ & 0.683772 & $B^{\prime} \supset B$ & 0.687924 \\
\hline & $R_{9}$ & $A^{\prime}$ & 0.683772 & $B^{\prime} \supset B$ & 0.687924 \\
\hline $\mathrm{mr} 4$ & & $A^{\prime}$ & 0.683772 & $B^{\prime} \supset B$ & 0.440355 \\
\hline $\mathrm{mr} 5$ & & $A^{\prime}$ & 0.683772 & $B^{\prime} \supset B$ & 0.440355 \\
\hline mr6 & & $A^{\prime}$ & 0.683772 & $B^{\prime} \supset B$ & 0.525030 \\
\hline $\mathrm{mr} 7$ & & $A^{\prime}$ & 0.683772 & $B^{\prime} \supset B$ & 0.437789 \\
\hline
\end{tabular}

sums method. Let $p^{(k)}$ be the peak value of $A^{(k)}$ and $h^{(k)}$ be the corresponding height of the clipped version of $A^{(k)}$. Then, the defuzzified value will be given by

$$
u^{*}=\frac{\sum_{k=1}^{m} p^{(k)} \cdot h^{(k)}}{\sum_{k=1}^{m} h^{(k)}} .
$$

Specificity, height and peak values are sometimes used simultaneously to compute defuzzified value. Here, we propose a method for the defuzzification of fuzzy sets as in the following:

$$
u^{*}=\frac{\sum_{k=1}^{m} p^{(k)} \cdot h^{(k)} \cdot s_{(k)}}{\sum_{k=1}^{m} h^{(k)} \cdot s_{(k)}} .
$$

Example 2. In this example, we consider two fuzzy sets as given in Figure 12 and corresponding clipped fuzzy sets in Figure 13. From these sets, we compute the defuzzified 


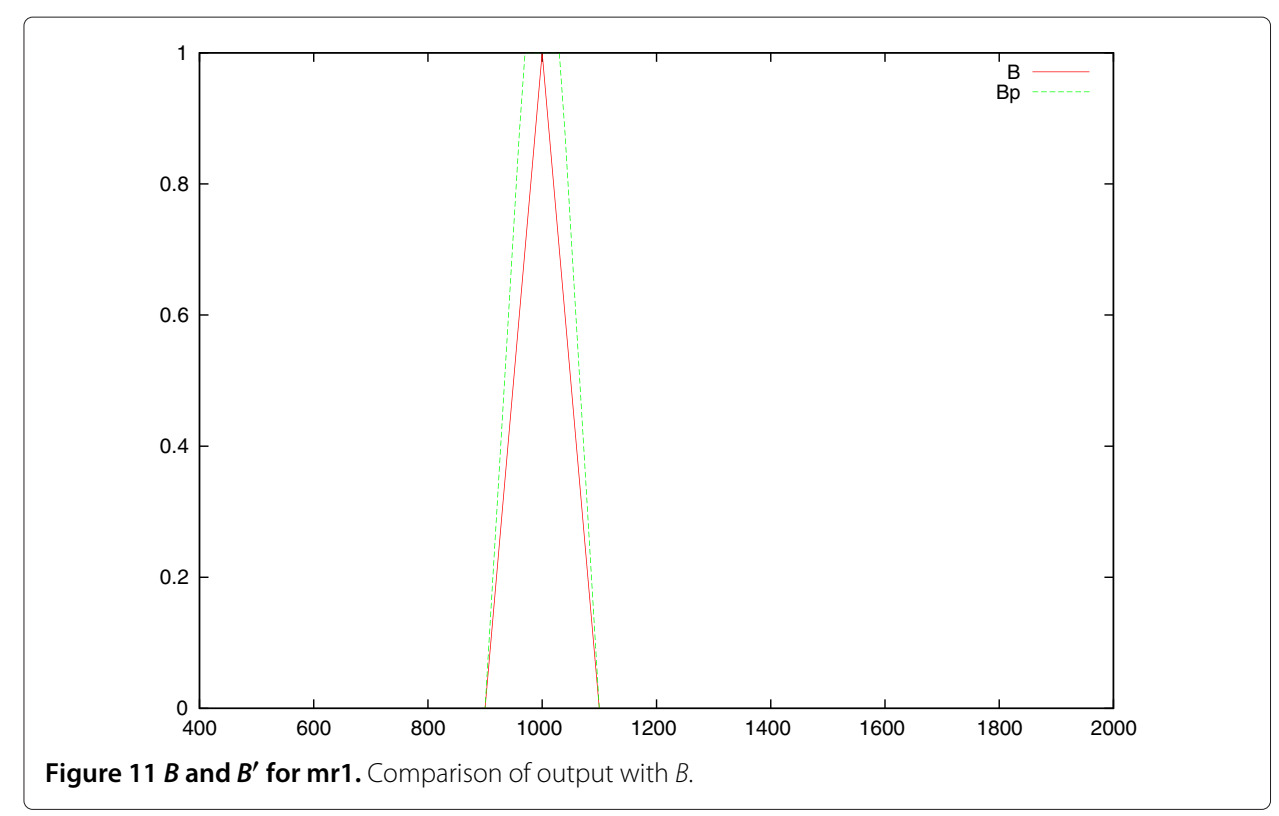

value of the fuzzy sets using the methods of defuzzification as given in (10), (11) and (12), respectively.

$\operatorname{Sp}\left(A^{(1)}\right)=0.175, \operatorname{Sp}\left(A^{(2)}\right)=0.275 ; p^{(1)}=3, p^{(2)}=4$ and $h^{(1)}=0.6, h^{(2)}=0.8$.

From (10), (11) and (12), we get $u^{*}=3.61, u^{*}=3.57$ and $u^{*}=3.67$.

We apply these three types of defuzzification methods in our fuzzy systems.

\section{Application in different models}

Let us consider a generalised model as presented in Table 7. This form of reasoning is used in rule-based fuzzy systems. In particular, it is used in pattern classification and fuzzy control. Let there be $n$ linguistic variables associated with another linguistic variable $Y$ according to the following $m$ fuzzy rules. The problem is to find the linguistic value of the variable $Y$ as suggested by the rules, when the values of the $n$ variables are given.

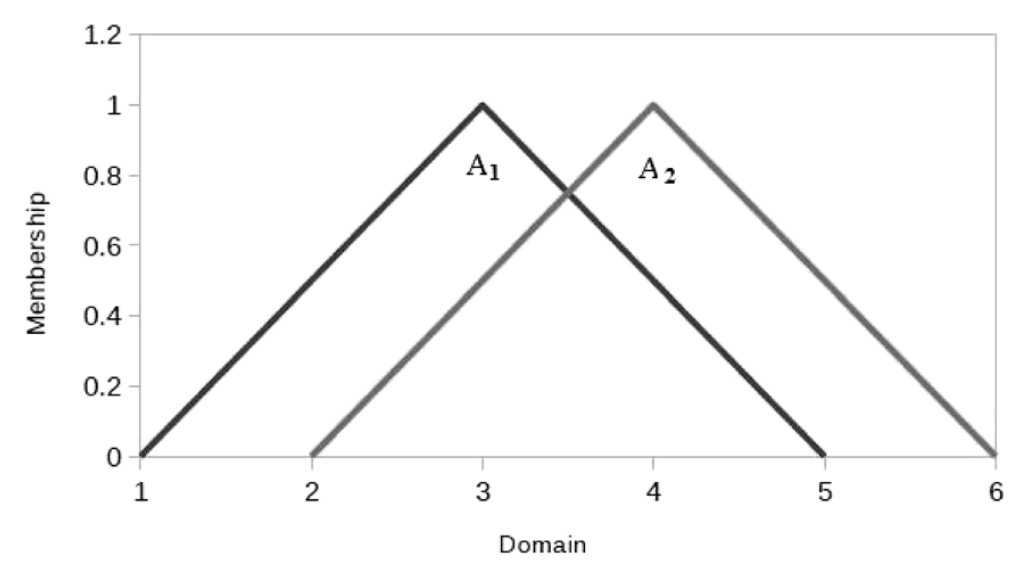

Figure 12 Fuzzy sets for defuzzification. 


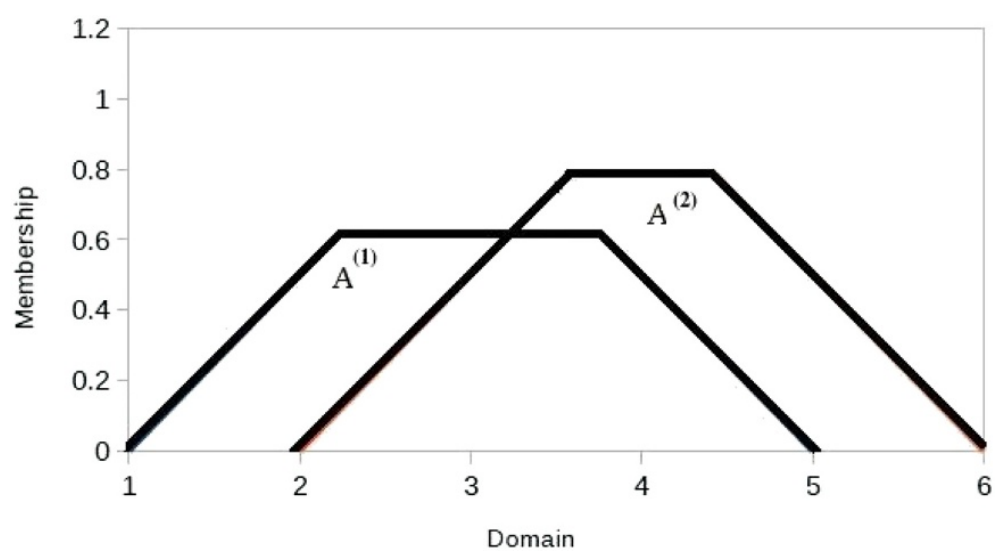

Figure 13 Clipped fuzzy sets for defuzzification.

Under the conventional technique, for each rule, the consequent fuzzy set is calculated according to existing method of inference as already described, and then the union of all consequent fuzzy sets is taken as the conclusion which is then defuzzified, if necessary, using some defuzzification scheme. In similarity-based reasoning, this cannot be done. Here, the membership values computed from the modified induced relation becomes less and less specific as the similarity between the facts and antecedent of a rule decreases. In conventional practice, the membership values of various elements become equal to the maximum, making it an ambiguous one (more alternatives with similar membership values at the positive level) with the reduction of the firing strength (used in deriving a conclusion), but the membership values at which the ambiguity occurs become less than one. For example, in case of Mamdani type of reasoning, if the firing strength of a rule is say 0.3 , then all alternatives which have membership values greater than or equal to 0.3 take membership values of 0.3 . On the other hand, in the present case, if the similarity value is 0.3 , then the membership values of elements in the inferred fuzzy set will be at least 0.3. Moreover, the elements having membership value greater than or equal to 0.3 in the consequent of the rule will be equal to one in the consequent fuzzy set. This means that with decrease in similarity the computed membership values increase and ultimately move close to the least specific case (with membership values of 1 for all alternatives). For this reason, we propose a new scheme, for computing the final conclusion, based on a measure of similarity. Our method is based on rule selection and then rule execution. In both cases, we use the concept of similarity between fuzzy sets as a basis of the task. For that, first of all, we compute $S\left(A_{i j}, A_{i}\right) ; i=1,2, \ldots, m$. Then, we perform the same

Table 7 Applicable form of approximate reasoning

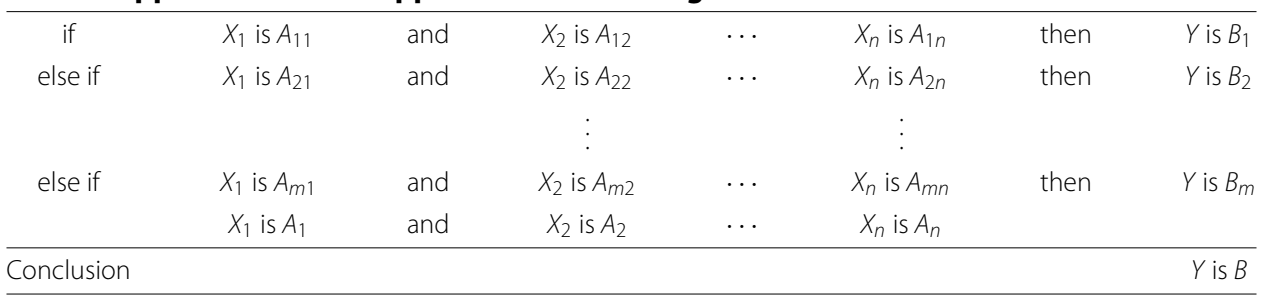


operation for different $j=1,2, \ldots, n$. Let $s_{i j}$ denote the different similarity values. We next compute the overall rule matching index from the above data as

$$
s^{i}=\min _{j} s_{i j}
$$

From among the $m$ distinct rules, we choose those rules for which $s^{i}>\epsilon$. This $\epsilon$ can be interpreted as a threshold in our case. We then apply the algorithm SEAR to generate a conclusion from each rule conformal for firing. The output can be generated using the intersection of fuzzy sets. It is important to note that the intersection operation is chosen in order to justify the rule selection procedure. Here, fewer rules are fired and the output of each rule is significant.

To compute $B^{\prime}$ as in Table 7, we apply the following algorithm.

\section{Algorithm AFSAR: applicable form of similarity-based approximate reasoning}

Step 1. Compute $s_{i j}$ for $i=1,2, \ldots, m ; j=1,2, \cdots, n$ and then $s^{i}$ according to (13).

Step 2. Define $\epsilon$ and find the rules conformal for firing.

Step 3. Translate the $i$ th rule, provided $s^{i}>\epsilon$, and compute the relation $R_{i}$ using any suitable translating rule possibly, a t-norm operator as given in Table 3.

Step 4. Modify $R_{i}$ with $s^{i}$ to obtain the modified conditional relation $R_{i}^{\prime}$ according to some modification scheme as given in Table 4.

Step 5. Use sup-projection operation on $R_{i}^{\prime}$ to obtain $B_{i}^{\prime}$ as given in (14).

$$
\mu_{B_{i}^{\prime}}(v)=\sup _{u_{1}, u_{2}, \ldots, u_{k}} \mu_{R\left(A_{1}\left|A_{i 1}, A_{2}\right| A_{i 2}, \ldots, A_{k}{ }^{\prime} \mid A_{i k}, B\right)}\left(u_{1}, u_{2}, \ldots, u_{k}, v\right) .
$$

Step 6. Compute the specificity measure of $B_{i}^{\prime}$ denoted by $\operatorname{Sp}\left(B_{i}^{\prime}\right)$, for all $i$ for which a rule is fired and set $\mathrm{Sp}=\max \operatorname{Sp}\left(B_{i}^{\prime}\right)$. If $\mathrm{Sp}>1-\epsilon(\epsilon>0)$, the predefined threshold, then output $B=\left\{\bigcap_{j} B_{j}^{\prime} \mid \operatorname{Sp}\left(B_{j}^{\prime}\right)>1-\epsilon\right\}$.

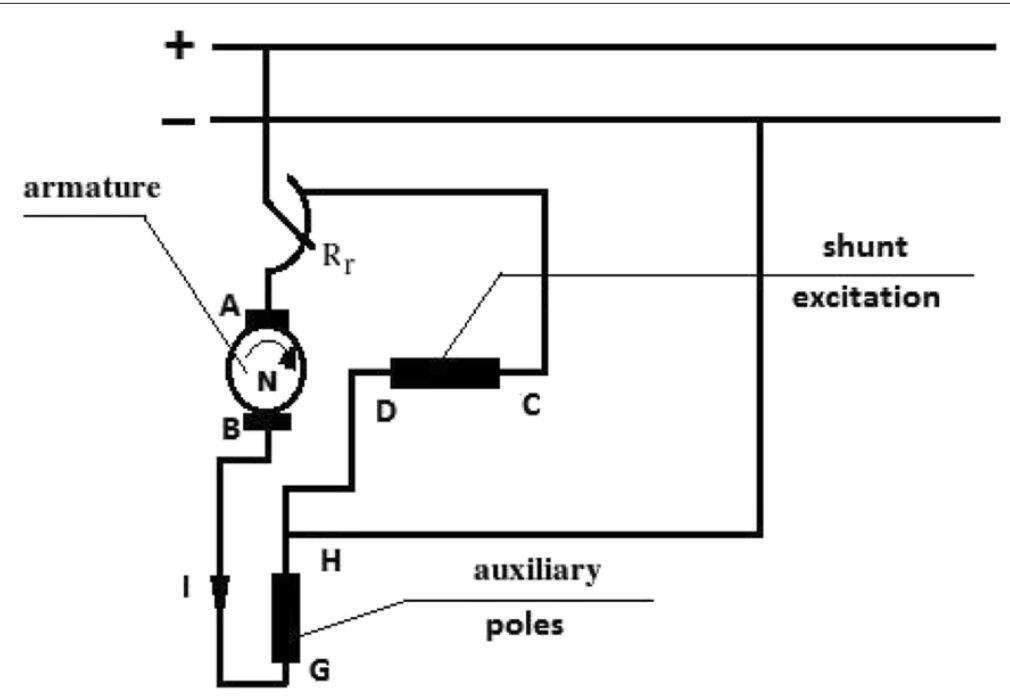

Figure 14 DC shunt motor. 
Table 8 Rule base of DC shunt motor

\begin{tabular}{|c|c|c|c|c|}
\hline $\mid \mathrm{f} /=A_{1}$ & then $N=B_{17}$ & also & If $/=A_{12}$ & then $N=B_{7}$ \\
\hline$|f|=A_{2}$ & then $N=B_{15}$ & also & If $/=A_{13}$, & then $N=B_{9}$ \\
\hline$|f|=A_{3}$ & then $N=B_{13}$ & also & If $/=A_{14}$, & then $N=B_{11}$ \\
\hline If $\mid=A_{4}$ & then $N=B_{11}$ & also & If $/=A_{15}$ & then $N=B_{13}$ \\
\hline$|f|=A_{5}$ & then $N=B_{9}$ & also & If $/=A_{16}$ & then $N=B_{15}$ \\
\hline$|f|=A_{6}$ & then $N=B_{7}$ & also & If $/=A_{17}$, & then $N=B_{17}$ \\
\hline$|\mathrm{f}|=A_{7}$ & then $N=B_{5}$ & also & If $/=A_{18}$ & then $N=B_{15}$ \\
\hline$|\mathrm{f}|=A_{8}$ & then $N=B_{3}$ & also & If $/=A_{19}$ & then $N=B_{13}$ \\
\hline$|f|=A_{9}$ & then $N=B_{1}$ & also & If $/=A_{20}$ & then $N=B_{11}$ \\
\hline If $/=A_{10}$ & then $N=B_{3}$ & also & If $/=A_{21}$, & then $N=B_{9}$ \\
\hline If $/=A_{11}$ & then $N=B_{5}$ & also & & \\
\hline
\end{tabular}

In the process, we find a conceptual change in similarity-based inference mechanism. A closer look at the connection between the proposed schemes and the existing schemes allows us to conclude that our schemes can be thought of as an integration of Zadeh's compositional rule of inference and similarity-based inference schemes. Such a scheme is expected to produce efficiency in inference mechanisms.

\section{A case study on DC shunt motor}

In this section, let us consider the DC shunt motor in Figure 14 as investigated in [33]. From the measurement of the current value $I$, the rotating speed value $N$ in steady states was determined by $N=f(I)$.

Human experts observed the behaviour of the DC shunt motor and described the relation between current and speed in the form of fuzzy conditional statements as in the following, where $I$ is the linguistic variable representing the motor current and $N$ is the linguistic variable representing the motor rotations.

Let the domain set for the variable $I$ be $U=\{0.0,0.1,0.2,0.3,0.4, \ldots, 9.9,10.0\}$. Let $A_{1}, A_{2}, A_{3}, A_{4}, \ldots, A_{21}$ be the fuzzy sets corresponding the points $0.0,0.5,1.0,1.5, \ldots, 10.0$. Let $V=\{400,410,420, \ldots, 1,990,2,000\}$ be the universe of discourse of the linguistic variable $N$. Let $B_{1}, B_{2}, \ldots, B_{17}$ be the fuzzy sets corresponding the points $400,500,600, \ldots, 2,000$. Now, we describe the behaviour of the motor (the specific relation between current and speed) using fuzzy rules as in Table 8 .

The data for the fuzzy model is given in Table 9, and the corresponding real static curve is given in Figure 15.

For a particular observed value of current expressed in natural language, we first translate the inexact concepts into fuzzy sets (the simple observation) or fuzzy relations (the complex rule) over the specified universe of discourse using triangular membership functions. We then perform approximate reasoning to obtain the corresponding speed of the DC motor using the algorithm SAR.

Table 9 Real data for DC shunt motor

\begin{tabular}{cccccccccccccc}
\hline $\boldsymbol{I}$ & $\boldsymbol{N}$ & $\boldsymbol{I}$ & $\boldsymbol{N}$ & $\boldsymbol{I}$ & $\boldsymbol{N}$ & $\boldsymbol{I}$ & $\boldsymbol{N}$ & $\boldsymbol{I}$ & $\boldsymbol{N}$ & $\boldsymbol{I}$ & $\boldsymbol{N}$ & $\boldsymbol{I}$ & $\boldsymbol{N}$ \\
\hline 0.0 & 2,000 & 1.5 & 1,400 & 3.0 & 800 & 4.5 & 600 & 6.0 & 1,200 & 7.5 & 1,800 & 9.0 & 1,600 \\
0.5 & 1,800 & 2.0 & 1,200 & 3.5 & 600 & 5.0 & 800 & 6.5 & 1,400 & 8.0 & 2,000 & 9.5 & 1,400 \\
1.00 & 1,600 & 2.5 & 1,000 & 4.0 & 400 & 5.5 & 1,000 & 7.0 & 1,600 & 8.5 & 1,800 & 10.0 & 1,200 \\
\hline
\end{tabular}






Figure 15 Real static characteristic of a DC motor.

The defuzzified input/output are plotted for a comparative assessment of the utility of the proposed similarity-based approximate reasoning methodology. The simulation results are presented in the following self-explanatory diagrams given in Figure 16.

\section{Conclusions}

Developing intelligent systems becomes necessary to handle modern computer-based technologies managing different kinds of information and knowledge. This paper discusses a theory to help provide solutions to difficult problems in the construction of intelligent systems in which the available information is supplied by human experts which

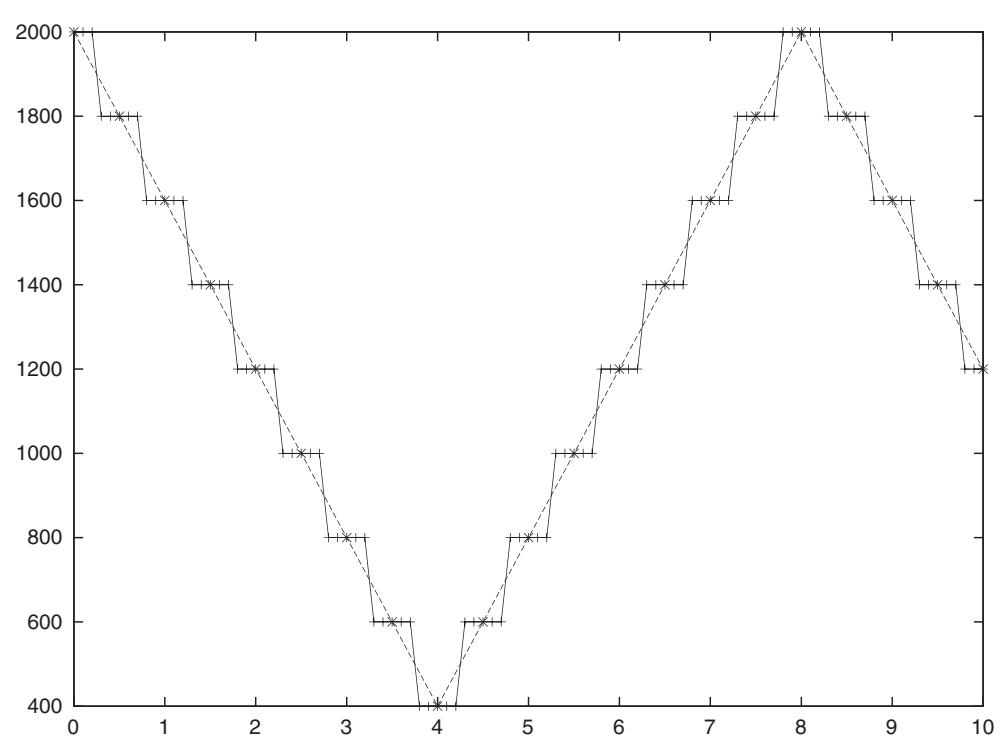

Figure 16 Comparison: real and proposed inference. 
at times are found incomplete, imprecise or even uncertain in nature and therefore inherently ambiguous. It is hoped that by upgrading existing methodologies through addition of concepts and techniques drawn from the fuzzy set theory opens the door to a substantial enhancement of our ability to model reality. In the process, we have developed a mechanism to compute the matching degree of two fuzzy sets - representation of imprecise concepts (e.g. low speed and very low speed of a DC motor).

It has been shown that the concept of similarity is inherent in approximate reasoning methodology. Different problems arising out of the use of existing compositional rule of inference as well as similarity-based reasoning have been discussed with suitable examples. Different functions used to measure the similarity between two inexact concepts are reviewed. We have proposed axioms to compute the similarity between two fuzzy sets and appropriately introduced the concept in approximate reasoning methodology. It may be argued that the proposed similarity-based approximate reasoning technique is a combination of Zadeh's compositional rule of inference and Turksen's similarity-based reasoning. It is shown that this method is a more general characterisation of similaritybased approximate reasoning, and Turksen's method is a special case of the proposed method.

We have suggested relevant issues involved in the design of fuzzy systems - introduced similarity in reasoning, similarity relation in fuzzification and the concept of specificity measure in defuzzification. The concept of similarity is effectively used in system control. It is hoped that the introduction of the specificity-based defuzzification technique will prove to be a powerful technique in qualitative control. Further research on the use of similarity-based approximate reasoning is necessary for a better understanding of the effect of the same on the cognitive process involved in qualitative modelling and simulation. Similarity-based reasoning is a basic mode of inference in fuzzy logic in a wide sense that provides a basis for formalisation of commonsense reasoning and a non-ad hoc computational framework for combining and deriving evidence in expert systems. Similarity-based approximate reasoning may be popularised because of the scope of its applications in wide and challenging fields of investigation.

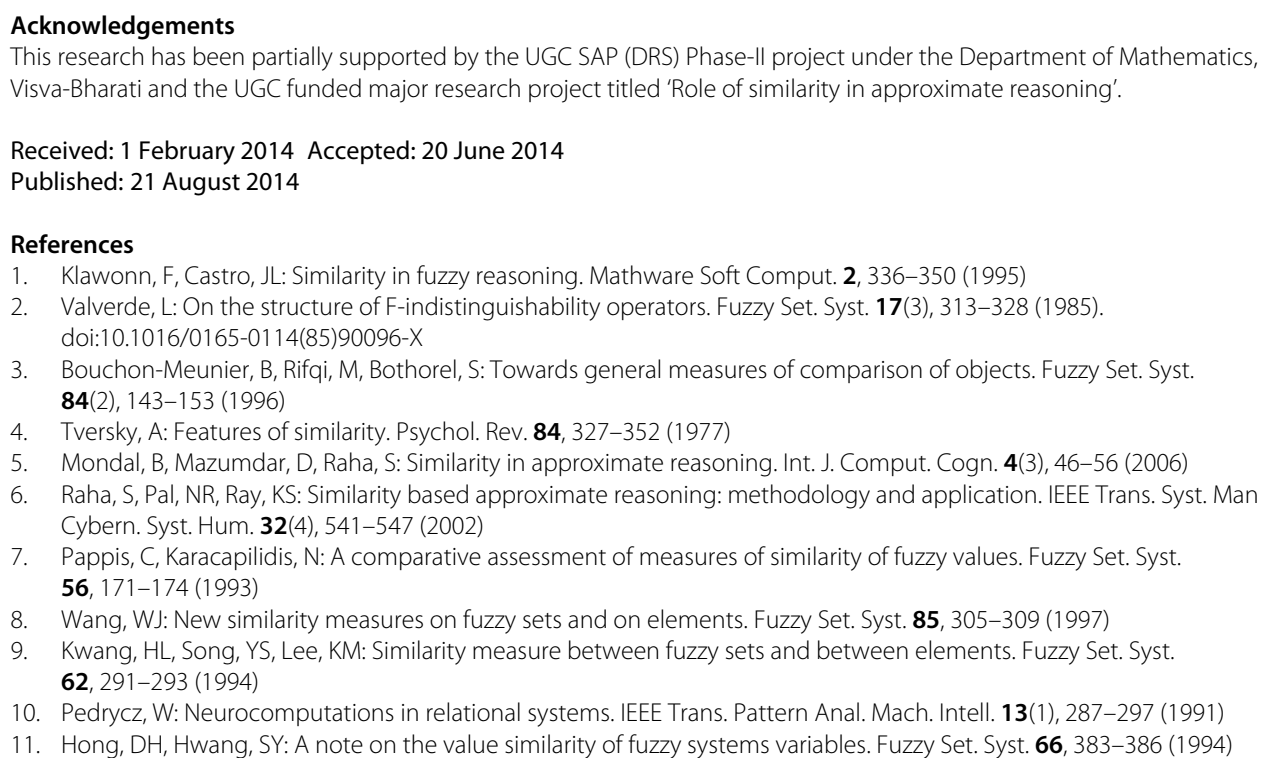


12. Zwick, R, Carlstein, E, Budescu, DV: Measures of similarity among fuzzy concepts: a comparative analysis. Inter. J. App. Reas. 1, 221-242 (1987)

13. Lesot, M-J, Rifqi, M, Benhada, H: Similarity measures for binary and numerical data: a survey. Int. J. Knowl. Eng. Soft Data Paradigms 1(1), 63-84 (2009)

14. Bouchon-Menuier, B, Rifqi, M, Lesot, M-J: Similarities in fuzzy data mining: from a cognitive view to real-world applications. In: Zurada, JM, Yen, GG, Wang, J (eds.) Computational Intelligence: Research Frontiers. Lecture Notes in Computer Science, vol. 5050, pp. 349-367. Springer, Berlin, (2008)

15. Zadeh, LA: A theory of approximate reasoning. In: Hayes, J, Michie, D, Mikulich, LI (eds.) Machine Intelligence vol. 9, pp. 149-194. Elsevier, New York, (1979)

16. Zadeh, LA: The concept of a linguistic variable and its application to approximate reasoning I, II. Inf. Sci. 8, 199-249301357 (1975)

17. Zadeh, LA: Outline of a new approach to the analysis of complex systems and decision processes. IEEE Trans. SMC 3, 28-44 (1973)

18. Turksen, IB, Zhong, Z: An approximate analogical reasoning approach based on similarity measures. IEEE Trans. SMC 18(6), 1049-1056 (1988)

19. Chen, SM: A new approach to handling fuzzy decision making problems. IEEE Trans. SMC 18, 1012-1016 (1988)

20. Tsang, FCC, Lee, JWT, Yeung, DS: Similarity-based fuzzy reasoning methods for fuzzy production rules. In: Proc. Sixth IFSA World Congress, pp. 157-160, Sao Paolo, (22-28 July 1995)

21. Yeung, DS, Tsang, ECC: A comparative study on similarity-based fuzzy reasoning methods. IEEE Trans. SMC - Part B: Cyber. 27(2), 216-227 (1997)

22. Esteva, F, Garcia, P, Godo, L, Rodrigue, R: A modal account of similarity-based reasoning. Int. J. Approximate Reas. 16(3-4), 235-260 (1997)

23. Esteva, F, Garcia, P, Godo, L, Rupsini, E, Valverde, L: On similarity logic and the generalised modus ponens. In: Proceedings of the Third IEEE Conference on Fuzzy Systems, vol. 2, pp. 1423-1427, Orlando, (26-29 June 1994)

24. Bouchon-Menuier, B, Valverde, L: Analogy relations and inference. In: Proceedings of the Second IEEE International Conference on Fuzzy Systems, vol. 2, pp. 1140-1144, San Francisco, (28 Mar-1 Apr 1993)

25. Bouchon-Menuier, B, Valverde, L: A fuzzy approach to analogical reasoning. Soft Comput. 3(3), 141-147 (1999)

26. Bouchon-Menuier, B, Delechamp, J, Marsala, C, Rifqi, M: Several forms of fuzzy analogical reasoning. In: Proceedings of 6th IEEE International Conference on Fuzzy Systems, FUZZ-IEEE'97, vol. 1, pp. 45-50, Barcelona, (1-5 July 1997)

27. Yeung, DS, Tsang, ECC: Improved fuzzy knowledge representation and rule evaluation using fuzzy petri nets and degree of subsethoods. Intell. Syst. 9(12), 1083-1100 (1994)

28. Turksen, IB, Tian, Y: Combination of rules or their consequences in fuzzy expert systems. Fuzzy set. Syst. 58(1), 3-40 (1993)

29. Chen, SM: A weighted fuzzy reasoning algorithm for medical diagnosis. Decis. Support Syst. 11, 37-43 (1994)

30. Raha, S, Hossain, A, Ghosh, S: Similarity based approximate reasoning: fuzzy control. J. Appl. Logic. 6(1), 47-71 (2008)

31. Dubois, D, Prade, H: A note on measures of specificity for fuzzy sets. Int. J. Gen Syst. 10, $279-283$ (1985)

32. Yager, RR: Measuring tranquility and anxiety in decision making: an application of fuzzy sets. Int. J. Gen Syst. 8, 139-146 (1982)

33. Goralczany, MB, Kiszka, JB, Stachowicz, MS: Some problems of studying adequacy of fuzzy models. In: Yager, RR (ed.) Fuzzy Set and Possibility Theory, pp. 14-31. Pergamon Press, New York, (1982)

doi:10.1186/s40467-014-0018-0

Cite this article as: Pal et al.: Similarity in fuzzy systems. Journal of Uncertainty Analysis and Applications 2014 2:18.

\section{Submit your manuscript to a SpringerOpen ${ }^{\circ}$ journal and benefit from:}

- Convenient online submission

- Rigorous peer review

- Immediate publication on acceptance

- Open access: articles freely available online

- High visibility within the field

- Retaining the copyright to your article

Submit your next manuscript at $\boldsymbol{\triangleright}$ springeropen.com 